\title{
Computer Simulations of the \\ Motion and Decay of Global Strings*
}

C. Hagmannt and P. Sikivie

Physics Department

University of Florida

Gainesville, FL 32611

\begin{abstract}
:
Computer simulations have been carried out of the motion and decay of global strings, including spectrum analysis of the energy stored in the scalar field which describes the global string and the radiated Nambu-Goldstone bosons. We simulated relaxing pieces of bent string and collapsing circular loops. We find, for the string sizes investigated, that the spectrum of field energy hardens rather than softens while the string decays into $\mathrm{Na}$. zbu-Goldstone radiation. We argue on theoretical grounds that this is indeed the most plausible general behaviour.
\end{abstract}

\section{DISCLAIMER}

This report was prepared as an account of work sponsored by an agency of the United States Government. Neither the United States Government nor any agency thereof, nor any of their employees, makes any h'arranty, express or implied, or assumes any legal liability or responsibility for the accuracy, completeness, or usefulness of any information, apparatus, product, or bility for the accuracy, completeness, or usefulness of any information, apparatus, process disclosed, or represents that its use would not infringe privately owned rights. Reference herein to any specific commercial product, process, or service by trade name, trademark, manufacturer, or otherwise does not necessarily constitute or imply its endorsene The views mendation, or favoring by the United States Government or any agency theer. The of the and opinions of authors expressed herein do not

* Work supported in part by the Department of Energy under contract DE-FG05-86ER40272.

$\dagger$ Present address: Physics Department, University of California, Berkeley, CA 94720 


\section{Introduction}

During the past decade, considerable effort has been devoted to the study of various topological objects which may appear in phase transitions in the early universe. ${ }^{1}$ Cosmic strings are particularly interesting because they could be present in the universe today as small regions of space which are hung up in a false vacuum of very high energy density. ${ }^{2}$ If one were to discover such strings, one would have the opportunity of learning some physics in an energy regime which is way out of reach of earth based laboratory experiments.

One possible type of cosmic string is the global string. Global strings are contained in particle physics models in which a global symmetry group $G$ breaks down spontaneously to a subgroup $H$ such that the first homotopy group $\pi_{1}(G / H)$ is nontrivial. The simplest example is given by the action

$$
S=\int d^{4} x\left[\frac{1}{2} \partial_{\mu} \varphi^{\dagger} \dot{v}^{\mu} \varphi-\frac{\lambda}{4}\left(\varphi^{\dagger} \varphi-v^{2}\right)^{2}\right]
$$

where $\varphi=\varphi_{1}+i \varphi_{2}$ is a complex scalar field. This model has a global U(1) symmetry which is broken spontaneously by the vacuum expectation value

$$
\varphi=v e^{i a / v}
$$

Here $a$ is the Nambu-Goldstone (NG) boson. In this example, a straight global string located on the $\hat{z}$-axis is the configuration

$$
\varphi(\rho, \theta)=v f(\rho) e^{i \theta}
$$

where $(z, \rho, \theta)$ are cylindrical coordinates. This configuration of infinite extent is topologically stable. The function $f$ is such that the energy per unit length

$$
\begin{aligned}
\tau & =\int d^{2} \mathrm{x}\left[\frac{1}{2} \vec{\nabla} \varphi^{\dagger} \cdot \vec{\nabla} \varphi+\frac{\lambda}{4}\left(\varphi^{\dagger} \varphi-v^{2}\right)^{2}\right] \\
& =\pi v^{2} \int_{0}^{\infty} \rho d_{\rho^{\prime}}\left[\left(\frac{d f}{d \rho}\right)^{2}+\frac{1}{\rho^{2}} f^{2}+\frac{\lambda v^{2}}{2}\left(f^{2}-1\right)^{2}\right]
\end{aligned}
$$


is minimized. Hence

$$
\frac{d^{2} f}{d \rho^{2}}+\frac{1}{\rho} \frac{d f}{d \rho}-\frac{f}{\rho^{2}}-\lambda v^{2}\left(f^{2}-1\right) f=0
$$

which is solved by $f(\rho) \equiv F(\rho / \delta)$ where $\delta=\frac{1}{\sqrt{\lambda v}}$ and $F$ is some function with the limiting behaviour

$$
\begin{aligned}
& F(\mathrm{x}) \rightarrow 1-\frac{1}{2 \mathrm{x}^{2}}+0\left(\frac{1}{\mathrm{x}^{4}}\right) \quad \text { as } \mathrm{x} \rightarrow \infty \\
& \rightarrow c \mathrm{x}+0\left(\mathrm{x}^{3}\right) \quad \text { as } \mathrm{x} \rightarrow 0 \text {. }
\end{aligned}
$$

Numerically, one finds that $\mathrm{c}$ is of order 0.6 . The region within a distance $\delta$ from the center of the string is called the core. Outside the core, the magnitude of $\varphi$ rapidly approaches $v$ and the main dynamics is in the massless degree of freedom $a$. The energy per unit length (1.4) is logarithmically divergent at large distances. Let L be a long distance cut-off. Then

$$
\tau \simeq \pi v^{2} \int_{\delta}^{L} \frac{d \rho}{\rho}=\pi v^{2} \ln (L / \delta)
$$

Eq. (1.7) gives the main contribution to $\tau$ provided $\ln (L / \delta)>>1$. It is the contribution due to the gradient in the NG field $a=v \theta$, outside the string core. Eq. (1.7) neglects the contribution of order $0(1) v^{2}$ from the core of the string. For cosmic global strings, a long distance cut-off is automatically provided because neighboring strings will have different directions, and hence their winding numbers will cancel out on average. Thus, for cosmic global strings, $L$ is of order the distance between strings which is typically of order the horizon size $t$ ( $t=$ time since the Big Bang). For $t \simeq 10^{10}$ years and $v \simeq 10^{14} \mathrm{GeV}$, we have $\ell n(L / \delta) \simeq \ell n(t v) \simeq 130$. For axion strings at $\mathrm{QCD}$ time, $t \simeq 10^{-6} \mathrm{sec}, v \simeq 10^{12} \mathrm{GeV}$ and hence $\ell n(t v) \simeq 70$. In either case, most of the energy is in the NG field outside the string core.

So far everything is straightforward. However, let us next consider a bent piece of string or a closed loop. It is expected that such an object will move and emit NG radiation. A 
closed loop will eventually disappear completely after having released all its energy into NG radiation. is bent string will straighten itself out by releasing its excess energy into NG radiation. The questions are

1) how much time does the decay process take?

2) what is the spectrum of the NG radiation?

There is disagreement in the existing literature what the right answers to these quesiions are likely to be. The following candidate answers have been put forth in the limit of large $\ln (L / \delta):$

A) the number of oscillations of the closed loop or bent string before it has lost most of its energy is of order $\frac{1}{10} \ln (L / \delta)$, where $L$ is the initial size, and the emitted NG radiation has wavelength of order $L . .^{3-6}$

B) the closed loop or bent string releases its excess energy at once, in the order of one oscillation time, and the emitted NG radiation has a $\frac{1}{k}$ power spectrum with a high wavevector cutoff of order $\delta^{-1}$ and a low energy cutoff of order $L^{-1} \cdot 7,8$

Of course, the right answer may very well be neither of the above.

The candidate answers A and B will be discussed in detail in section II. For the time being, let us simply note that they both have a certain self-consistency. In scenario $A$, the string radiates energy mainly at a frequency of order its inverse size, which is also its oscillation frequency. If one increases $\ln (L / \delta)$ by decreasing $\delta$ keeping $L$ fixed, the radiating power $P$ does not change but the total energy $E$ stored in the string increases like $\ln (L / \delta)$. Hence the number of oscillations before the string has decayed away goes as $\ln (L / \delta)$. In scenario $\mathrm{B}$, the string radiates its energy at all frequencies at once, both $P$ and $E$ are of order $\ln (L / \delta)$ and the number of oscillations after which the string has decayed away is of order one. In this short time, the spectrum of the energy stored in the NG field cannot change much. It is clear from (1.7) that a straight string has a $\frac{d E}{d k} \sim \frac{1}{k}$ 
energy spectrum with a high wavevector cutoff of order $\delta^{-1}$ and a low wavevector cutoff of order $L^{-1}$. Bending the string does not change the shape of the $\frac{1}{k}$ spectrum except at wavevectors of order $L^{-1}$. Of course, the amplitude of the $\frac{1}{k}$ spectrum is proportional to the total length of the string. If the spectrum is time independent, the radiated NG bosons must have a $\frac{1}{k}$ spectrum as well, with an amplitude proportional to the difference in lengths of the initial and final strings.

One might thus say that in scenario A, the global string is "underdamped" in the limit where the ratio $\ell n(L / \delta)$ of field energy to core energy is large. Whereas, in scenario $B$, the global string is "critically damped" in that same limit. It is certainly an oversimplification to use the words "underdamped" and "critically damped" in this context since the decay of a global string is a much more complex process than the dissipation of energy in a simple harmonic oscillator. However at present our understanding of the decay of a global string is sufficiently poor that the words in question are adequate to describe the range of our conjectures. The question might then be asked: could the global string be overdamped? In fact, Gradwohl et al. ${ }^{9}$ have pointed out that the motion of vortices in superfluid ${ }^{4} \mathrm{He}$, which are the closest thing to cosmic global strings we have available in the laboratory, is very much overdamped. However the reason for this overdamping can be found in the interaction of the vortices with the background medium. Because of this interaction, the dynamics of the vortices' motion is not invariant under Lorentz or Galilean boosts. On the other hand, it should be emphasized that we do not know for certain that Lorentz invariance is exact at the high energy scale $v$ either. If global strings exist in the universe today, the phenomena occurring near or in their core would probe physics at energy scales much higher than any which have been explored thus far. If Lorentz invariance is broken at those high energy scales, the global strings are quite likely to be overdamped as suggested by the behaviour of vortices in superfluid ${ }^{4} H e$.

We assume in this paper that Lorentz invariance is not broken. Also, our computer sim- 
ulations are only of the m’del of Eq. (1.1). There is however another representation $10,11,4$ of a global string, which is thought to be equivalent to the model of Eq. (1.1) in its essential features:

$$
S^{\prime}=\mu_{0} \int d^{2} \sigma \sqrt{-\operatorname{det}\left(h_{a b}\right)}+\frac{1}{12} \int d^{4} x H_{\mu \alpha \beta} H^{\mu \alpha \beta}+\frac{g}{2} \int d^{2} \sigma A_{\alpha \beta} \partial_{a} \mathrm{X}^{\alpha} \partial_{b} \mathrm{X}^{\beta} \epsilon^{a b}
$$

The first term is the Nambu-Goto action for the string core. Thus

$$
h_{a b}=\eta_{\mu \nu} \partial_{a} \mathrm{X}^{\mu} \partial_{b} \mathrm{X}^{\nu} \quad a, b=0,1
$$

where $\eta_{\mu \nu}$ is the space-time metric, here assumed to be Minkowskian, and $\mathrm{X}^{\mu}(\sigma, \tau)$ is the location of the world-surface of the string core in space-time; $\sigma^{0}=\tau, \sigma^{1}=\sigma$ and $\partial_{a}=\frac{\partial}{\partial \sigma^{a}} . \mu_{0}$ is the energy per unit length in the string core. The thickness of the latter is neglected. If we represent the global string of Eq. (1.3) by $S^{\prime}$ we must have $\mu_{0}=0(1) v^{2}$. The second term of $S^{\prime}$ is the kinetic term for the Kalb-Ramond ${ }^{10}$ field $A_{\alpha \beta}(\mathrm{x})$ which is an anti-symmetric $\left(A_{\alpha \beta}=-A_{\beta \alpha}\right)$ gauge field. We have

$$
H_{\mu \alpha \beta} \equiv \partial_{\mu} A_{\alpha \beta}+\partial_{\alpha} A_{\beta \mu}+\partial_{\beta} A_{\mu \alpha}
$$

$S^{\prime}$ is invariant under the gauge transformations

$$
A_{\alpha \beta} \rightarrow A_{\alpha \beta}+\partial_{\alpha} \Lambda_{\beta}-\partial_{\beta} \Lambda_{\alpha}
$$

where $\Lambda_{\alpha}(\mathrm{x})$ is an arbitrary vector field. The Kalb-Ramond field describes a single dynamical degree of freedom. This is because three (the $A_{0 i}, i=1,2,3$ ) of the six independent components $A_{\alpha \beta}$ have no canonical momenta and hence are variables of constraint. The remaining degrees of freedorn $\vec{A}=\hat{i} \epsilon_{i j k} A_{j k}$ transform under (1.11) as $\vec{A} \rightarrow \vec{A}+\vec{\nabla} \times \vec{\Lambda}$. Hence only the longitudinal part of $\vec{A}$ is a true dynamical degree of freedom. It was pointed out by Witten ${ }^{11}$ that the NG field a and the Kalb-Ramond field $A^{\alpha \beta}$ are dual to each other:

$$
H_{\mu \nu \lambda}=\epsilon_{\mu \nu \lambda \beta} \partial^{\beta} a
$$


with the global string core playing the role of source for the $A^{\alpha \beta}$ fie!d. The interaction of $A_{\alpha \beta}(\mathrm{x})$ with the global string core is described by the last term of $S^{\prime}$

$$
S_{i n t}^{\prime}=\int d^{4} \mathrm{x} \frac{1}{2} A_{\alpha \beta}(\mathrm{x}) j^{\alpha \beta}(\mathrm{x})
$$

where

$$
j^{\alpha \beta}(\mathrm{x})=g \int d^{2} \sigma \delta^{4}(\mathrm{x}-\mathrm{X}(\sigma, \tau)) \epsilon^{a b} \partial_{a} \mathrm{X}^{\alpha} \partial_{b} \mathrm{X}^{\beta}
$$

is the current carried by the global string core. $S_{\text {int }}^{\prime}$ enforces the topological constraint that the angle $\alpha(\mathrm{x})=a(\mathrm{x}) / v$ change by $2 \pi$ when $\mathrm{x}$ moves around the string core once, provided (see below) that

$$
g=2 \pi v
$$

The equations of motion which follow from $S^{\prime}$ are

$$
\begin{gathered}
\partial_{\mu} H^{\mu \alpha \beta}(\mathrm{x})=j^{\alpha \beta}(\mathrm{x}) \\
\mu_{0} \frac{\partial}{\partial \sigma^{a}} \sqrt{-\operatorname{det}\left(h_{c d}\right)} h^{a b} \frac{\partial X^{\mu}}{\partial \sigma^{b}}=\frac{g}{2} H_{\mu \alpha \beta} \epsilon^{a b} \partial_{a} X^{\alpha} \partial_{b} X^{\beta}
\end{gathered}
$$

where $\left(h^{a b}\right)=\left(h_{a b}\right)^{-1}$. The action (1.8) is invariant under arbitrary reparametrizations $(\sigma, \tau) \rightarrow\left(\sigma^{\prime}(\sigma, \tau), \tau^{\prime}(\sigma, \tau)\right)$ of the world-surface of the string core. It is possible to choose $(\sigma, \tau)$ such that

$$
\left(h_{a b}\right)=\sqrt{-\operatorname{det}\left(h_{c d}\right)}\left(\begin{array}{cc}
1 & 0 \\
0 & -1
\end{array}\right) .
$$

This is called the conformal gauge. In addition, using (1.11), one can adopt the Lorentz gauge $\partial_{\mu} A^{\mu \nu}=0$. In the combined conformal-Lorentz gauge, Eqs. (1.16) become

$$
\begin{gathered}
\partial_{\mu} \partial^{\mu} A^{\alpha \beta}(\mathrm{x})=g \int d^{2} \sigma \epsilon^{a b} \partial_{a} X^{\alpha} \partial_{b} X^{\beta} \delta^{4}(\mathrm{x}-X(\sigma, \tau)) \\
\mu_{0}\left(\frac{\partial^{2}}{\partial \tau^{2}}-\frac{\partial^{2}}{\partial \sigma^{2}}\right) X^{\mu}=\frac{g}{2} H^{\mu \alpha \beta} \epsilon^{a b} \partial_{a} X_{\alpha} \partial_{b} X_{\beta}
\end{gathered}
$$

For a straight string along the $\mathrm{z}$-axis $(\tau=t, \sigma=z)$, the solution is

$$
X^{\mu}(t, z)=(t, 0,0, z)
$$




$$
A^{03}=-\frac{g}{2 \pi} \ln \rho
$$

Eq. (1.20) is consistent with Eq. (1.12) and $a=v \theta$ provided $g=2 \pi v$. Note however that the short distance cutoff $\delta$ must introduced by hand into the model of Eq. (1.8) because the core has zero thickness in that model and hence the static field energy per unit length is logarithmically divergent. We will need the formalism of Eqs. (1.8-1.20) as background information when discussing in section II some of the theoretical ideas which have been put forth concerning the motion and decay of global strings.

The issue how a global string moves and decays and what is the spectrum of NG radiation emitted in the decay process is relevant to the estimate of the present cosmological axion energy density. Indeed, cosmic axion strings may be an important source of cosmic axions. ${ }^{3,7}$ The axion strings appear in the early universe during the phase transition in which the $U_{P Q}$ (1) quasi-symmetry of Peccei and Quinn gets spontaneously broken. This phase transition occurs at a temperature $T_{P Q}$ of order the magnitude $v_{a}$ of the vacuum expectation value which spontaneously breaks $U_{P Q}(1)$. The cosmic axion strings disappear at the QCD phase transition $\left(T_{1} \sim 1 \mathrm{GeV}\right.$ and $t_{1} \sim 10^{-6}$ sec $)$ at which time they become the edges of axion domain walls. The evolution of these domain walls has been discussed elsewhere. ${ }^{12,13}$ Assuming that no inflation takes place between $T_{P Q}$ and $T_{1}$, axion strings are present between the two phase transitions with a density of order one string per horizon. The axion strings dissipate their energy into axion radiation. One finds that, at cosmic time $t$, the total number density of axions that have been radiated by cosmic axion strings is 7

$$
n_{a}^{s t r}(t) \simeq \frac{1}{t^{3 / 2}} \int_{t_{P Q}}^{t} \frac{d t^{\prime}}{t^{\prime 3 / 2}} \frac{\tau\left(t^{\prime}\right)}{\bar{\omega}\left(t^{\prime}\right)}
$$

where $\tau(t)=\pi v_{a}^{2} \ln \left(t / \delta_{a}\right)$ is the energy per unit length of the axion string and

$$
\frac{1}{\bar{\omega}(t)} \equiv \int \frac{d E}{d \omega}(t) \frac{d \omega}{\omega} / \int \frac{d E}{d \omega}(t) d w .
$$


Here, $\frac{d E}{d \omega}(t)$ is the energy spectrum of axions radiated at time $t$. The axions that are radiated at time $t$ are radiated by axion strings which are initially bent over a distance scale of order $\mathrm{t}$ and which are relaxing to lower energy configurations. If the spectrum of axions radiated by such strings is peaked at wavevectors of order $\frac{2 \pi}{t}$ (scenario $A$ ) then $\bar{\omega}(t) \simeq \frac{2 \pi}{t}$ and

$$
n_{a}^{s t r}\left(t_{1}\right) \simeq \frac{v_{a}^{2}}{t_{1}} \ln \left(t_{1} / \delta_{a}\right) .
$$

On the other hand, in scenario $B$, where the spectrum of radiated axions is $\frac{d E}{d \omega} \sim \frac{1}{\omega}$ with a low wavevector cutoff of order $t^{-1}$ and a high wavevector cutoff of order $\delta_{a}^{-1}$, we have $\bar{\omega}(t) \simeq \frac{2 \pi}{t} \ln \left(t / \delta_{a}\right)$ and hence

$$
n_{a}^{s t r}\left(t_{1}\right) \simeq \frac{v_{a}^{2}}{t_{1}}
$$

We can compare Eqs. (1.23) with the rumber density of cosmic axions produced by initial vacuum misalignment ${ }^{14}$

$$
n_{a}^{v a c}\left(t_{1}\right) \simeq \frac{v_{a}^{2}}{N_{a}^{2}} \frac{1}{t_{1}}
$$

where $N_{a}$ is an integer which expresses the QCD anomaly of the $U_{P Q}(1)$ current and which, in most cases, equals the number of degenerate vacua of the axion model. ${ }^{12}$ The requirement that the cosmological axion energy density from initial vacuum misalignment does not overclose the universe imposes the constraint

$$
\frac{v_{a}}{N_{a}} \lesssim 10^{12} \mathrm{GeV} \quad \text { or } \quad m_{a} \gtrsim 10^{-5} \mathrm{eV}
$$

under the assumption, which is satisfied in most cosmological scenarios, that the atio of the axion number density to the entropy density remains constant from QCD time till the present.

Let us first ascume that $N_{a}=1$. In some sense, $N_{a}=1$ is favored over $N_{a} \neq$ 1 because $N_{a}=1$ axion models have a particularly straightforward mechanism to rid the early universe of axion domain walls. ${ }^{13}$ This is an important consideration since we 
are implicitly assuming, for cosmic axion strings to be relevant at all, that there is no inflation after the Peccei-Quinn phase transition. In scenario A, one would conclude that the cosmological bound on the axion mass from axions radiated by cosmic axion strings is roughtly a factor $\ell n\left(t_{1} / \delta\right) \approx 70$ times more severe than the bound from initial vacuum misalignment [compare Eqs. (1.23a) and (1.24)]. In that case there may be little or no room left between the cosmological bound and the bound from the supernova $\left(m_{a} \lesssim 10^{-3} \mathrm{eV}\right) .^{15}$ In scenario $\mathrm{B}$, on the other hand, one would conclude that the cosmological bound on the axion mass from axions radiated by cosmic axion strings is roughly of the same order of magnitude as the bound from initial vacuum misalignment [compare Eqs.(1.23b) and $(1.24)]$.

Note however that there is at least a possibility that $N_{a} \neq 1$. One can rid the early universe of axion domain walls in this case by introducing a tiny explicit breaking of the $U_{P Q}(1)$ symmetry which slightly lowers one of the $N_{a}$ vacua with respect to the others. ${ }^{12}$ A small bias of this kind can eliminate the domain walls from the early universe yet be compatible with the Peccei-Quinn solution to the strong CP problem. Comparison of Eqs. (1.23b) and (1.24) shows that if $N_{a}$ is large, the cosmolugical axion energy density from cosmic axion strings will dominate over that from initial vacuum misalignment even in scenario $B$. The last three paragraphs can be summarized as follows: if there is no inflation after the Peccei-Quinn phase tzansition, cosmic axion strings make a large - possibly the largest - contribution to the cosmic axion energy density. The size of the contribution depends upon the shape of the spectrum of NG bosons radiated by global strings.

Additional motivation for the study of the motion and decay of global strings is provided by recent attempts by one of us (P.S.) to interpret certain observational data as evidence for the existence of a massless Nambu-Goldstone boson and for the presence of cosmic global strings in the universe today. ${ }^{16}$ The cosmic global strings are postulated in accordance with conjecture B above - to deray into NG radiation with a $1 / \mathrm{k}$ spectrum. 
Some of the NG bosons convert to photons in the magnetic fields of galaxies, of clusters of galaxies and of astrophysical jets, through a mechanism that was first proposed as a means to detect on earih the axions emitted by the sun. ${ }^{17}$ It was argued that emission of NG radiation by cosmic global strings with subsequent conversion of the NG bosons into photons in the magnetic fields of various astrophysical objects may account for a number of puzzling observations: the occurrence of very high energy $\left(E>10^{9} \mathrm{GeV}\right)$ cosmic rays, the x-ray luminosity of astrophysical jets and the $90 \mathrm{deg}$ swings in the direction of the polarization vectors in radio-maps of astrophysical jets.

The remainder of the paper is organized as follows. In section II, we discuss the various theoretical ideas that have been put forth concerning the motion and decay of global strings. In section III we will describe the numerical techniques we have used to simulate global strings. In section IV, we will present our results for the motion and radiation spectrum of circular strings loops. In section V, we will do the same for bent strings. In section VI, we will summarize our results and conclude.

\section{Theoretical Discussion}

At present, there is no analytical treatment of the motion and decay of global strings which unequivocally answers the questions raised in the previous section. However, several approaches have been adopted and ideas put forward. It is our purpose in this section to give an account of these and to point out, in each case, whore the proposed treatments fail to be rigorous.

Let us first consider the arguments that lead to scenario A. Although the energy per unit length of a straight global string is logarithmically divergent at long distances, it is true nonetheless that the energy of the string is strongly localized near the core in the following sense: the fraction $f$ of energy which is located within a distance $d$ of the core is related to $d$ by $d=L\left(\frac{\delta}{L}\right)^{1-f}$ where $L$ is the long distance cutoff. Consider, for example, a global 
cusmic string in the present universe: $L \simeq t_{0} \simeq 10^{10}$ years, $\delta \simeq\left(10^{13} \mathrm{GeV}\right)^{-1}, \delta / L \simeq 10^{-50}$ and therefore $90 \%$ of the energy of the string is within a distance $d=10^{-5} L$ of the string core. From this point of view, a global string appears very much as a line-like object. One may thus expect that its motion is described in good approximation by the Nambu-Goto action. Following Davis ${ }^{3}$ and Vilenkin and Vachaspati ${ }^{4}$, let us assume that this is so. For a given Nambu-Goto motion, one can calculate how much power is radiated by the string. From dimensional analysis, the power radiated is

$$
P=\kappa v^{2}
$$

where $\kappa$ is a dimensionless number which depends upon the Nambu-Goto trajectory considered. Vilenkin and Vachaspati ${ }^{4}$, using Eq. (1.18a), evaluated $\kappa$ for a family of previously known closed loop solutions to the Nambu-Goto equations of motion. For this family, $\kappa$ varies over the approxirnate range 30 to 100 . Assuming that the family investigated is representative of the artual motions of cosmic global string loops, Vilenkin and Vachaspati conclude that the latter oscillate $n$ times before having half decayed away with $n$ given by

$$
n \simeq \frac{E}{P L} \simeq \frac{\tau}{\kappa v^{2}}=\frac{\pi}{\kappa} \ln (L / \delta) \sim \frac{1}{10} \ln (L / \delta)
$$

in agreement with an earlier estimate by R. Davis. Vilenkin and Vachaspati also found that the spectrum of the emitted NG radiation typically has a power spectrum $\frac{d P}{d k} \sim k^{-4 / 3}$ for large $k$. The emission of considerable amounts of power at large wavevectors is associated in this analysis with the appearance of cusps on the string loops for the family of NambuGoto motions that the authors used. These cusps are also responsible for the fact that $\kappa$ is much larger thas one. How generic these cusps are is not addressed. At any rate, although a. $\frac{d P}{d k} \sim k^{-4 / 3}$ spectrum has a lot of power at large wavevectors, it still leads to estimate (1.23a) rather than (1.23b) for the axion cosmological number density from cosmic axion strings. 
The striking feature of this analysis of the decay of a global string is that it predicts that the string becomes more and macre long-lived as the ratio $\ln (L / \delta)$ of field energy to core energy is increased. The reason given is that, when $\delta$ is made smaller, the radiated power $P$ does not increase because the moving string emits radiation only at wavelengths of order $L$, whereas the stored energy increases as $\ln (L / \delta)$. Note that, as was pointed out by Vilenkin and Vachaspati, the approximation of describing the string motion by the Nambu-Goto equations becomes self-consistent in the limit $\ell n(L / \delta) \rightarrow \infty$ in the following sense. In their analysis, the fraction of string energy radiated per oscillation period gots as $(\ell n L / \delta)^{-1}$. As a result, the hack-reacion effects due to the emission of radiation become small relative to the effects due to the string tension in the large $\ln (L / \delta)$ limit. This suggests that the Nambu-Goto equations of motion will be more and more exactly satisfied.

However, the self-consistency of an approximation does not constitute a definite proof of its validity. An alternative scenario for the motion and decay of the global string is that its motion on the scale $L$ of the string size is accompanied by motion on all scales between $L$ and $\delta$. In short, there could be "turbulence". For a hydrodynamic system, it is usually seif-consistent to assume that the flow will be + in-turbulent and to base one's further considerations on this hypothesis. But in actuality the flow can be either turbulent or non-turbulent. At the risk of appearing pedantically insistent, let us give an example. Consider a flag, i.e. a tivin piece of cloth held fixed at one straight boundary in a homogeneous flow of air. The syrumetry of this system suggests that the flag will lie in the plane determined by the fixed straight boundary and the direction of the homogeneous air flow, and it is self-consistent to assume that it will do so. But we know that under a large set of circumstances, the flag will flap instead. A simple reazon one can give for the widespread occurrence of turbulence is that entropy increases more rapidly in turbulent flow than in smooth flow. In other words, there are many circumstances nder which 
smooth flow oncupies an infinitesimally small part of phase-space.

Let us ask how much turbulence is required for the global string to radiate its stored energy at all frequencies at once. The power radiated by turbulent oscillations of the string core of frequency $\omega$ and amplitude $d_{\omega}$ is

$$
P_{\omega}=\kappa^{\prime} v^{2} d_{\omega}^{2} \omega^{3} L
$$

where $\kappa^{\prime}$ is another coefficient of order one. Note that $P_{\omega}$ is proportional to $L$ whereas the power $P$ of Eq. (2.1) is independent of $L$. For the global string to radiate its stored energy at all frequencies at once, it is sufficient that $P_{\omega} \simeq P$ for all $\omega$, or

$$
d_{\omega} \simeq \frac{1}{\omega(\omega L)^{\frac{1}{2}}}
$$

where we have neglected $\kappa$ and $\kappa^{\prime}$. The energy stored in the postulated oscillations of frequency $\omega$

$$
E_{\omega} \simeq \pi v^{2} \ln \left(\frac{1}{\omega \delta}\right) \omega^{2} d_{\omega}^{2} L \simeq \pi v^{2} \ln \left(\frac{1}{\omega \delta}\right) \frac{1}{\omega}
$$

is only a fraction $\frac{1}{\omega L} \ln \left(\frac{1}{\omega \delta}\right)$ of the energy of order $\pi v^{2} L$ stored in field modes with wavevectors of order $\omega$. It is entirely plausible that those modes will drive the string core in such a way that their energy is quickly released.

Another reason why scenario A may fail to be correct is that an oscillating loop can self-intersect with reconnection of the intersecting pieces. Through this mechanism, the loop cuts itself into two daughter loops. This is a process which can continue indefinitely. Let us assume now, for the sake of argument, that turbulence can be neglected entirely and that a loop of size $L$ emits radiation only of frequency $\frac{1}{L}$. On the other hand, let us assume that each loop has a non-zero probability $p$ at each oscillation to self-intersect with reconnection producing two daughter loops of roughly equal size. It is easy to see that, provided $p>(\ln L / \delta)^{-1}$, the total spectrum of Nambu-Goldstone radiation produced by the original loop, its two daughters, four granddaughters, etc... is $\frac{d E}{d \ell k} \sim$ constant, which is the same as the initial spectrum of field energy stored in the global string. 
We have thus found two mechanisms, turbulence and self-intersections, that may independently invalidate the assumptions underlying scenario A. But there is a general argument why scenario $\mathrm{A}$ is in a statistical sense unlikely. Indeed, in a classical field theory the number of degrees of freedom up to wavevector $k$ increases with $k$ as $k^{3}$, i.e. $\frac{d n}{d k} \sim k^{2}$ for the density of modes in $k$-space. The initial spectrum of field energy stored in a global string is $\frac{d E}{d k} \sim \frac{1}{k}$. Generally speaking, the statistical effect of interactions in a classical system is to distribute energy equally over all modes. For the classical field theory describing the global string, this would ultimately ; ield $\frac{d E}{d k} \sim k^{2}$ if thermal equilibrium were ever reached. Because the thermal spectrum is much harder than the initial spectrum, it may reasonably be expected that the effect of interactions during the motion of the global string will be to harden the spectrum. But scenario A proposes instead that the spectrum softens. It is asy to find proces ses which invalidate the assumptions of scenario A because the overall effect of a process is usually determined by considerations of phase space and, in the case of a global string, phase space definitely favors a hardening of the initial $\frac{d E}{d k} \sim \frac{1}{k}$ spectrum. Note however that scenario $\mathrm{A}$ is not exclv ded by the $2^{d}$ law of thermodynamics, at least not in an obvious way. The point is that the initial string field configuration has very low entrop $y$ since it is given by the initial position and transverse velocity of the string core which is a lower dimensional object. Thus, the entropy of the initial $\frac{1}{k}$ spectrum of ficld energy of a global string is much lower than the entropy of an arbitrary $\frac{1}{k}$ spectrum. So, the softening of the spectrum in scenario $A$ does not obviously imply a decrease in entropy. However, as emphasized above, it does require that something rather special happens which may be easily upset.

It is interesting to note in this regard that simulations of gauge strings have shown that they develop much more structure on small scales ${ }^{18}$ than had been previously expected. The small scale structure forms because intersections with reconnection of the strings cause small loops to be chopped off and produce kinks on long strings. The production 
of small scale structure on gauge strings obviously increases the entropy of a network of such strings. For a gauge string, which is a one-dimensional object, the density of modes is $\frac{d n}{d k} \sim$ constant, which is less weighted towards high wave-vectors than the $\frac{d n}{d k} \sim k^{2}$ density of modes of the field theory describing globai strings. From this point of view, one should expect entropy producing processes to be even more important for global strings than they are for local strings.

At any rate, we found mechanisms (turbulence or a process of self-intersections) which allow the energy stored in initial non-straight global string configurations to be released on all wavevectors scales more or less equally. It has not been proven yet that these processes do occur but it is reasonable to assume that they will. If they do occur, one obtains scenario $\mathrm{B}$ for the decay rate and radiation spectrum of global strings. ${ }^{7}$ Indeed, the extra dissipative processes will allow the string to decay in a time of order $L$, instead of $L \ln (L / \delta)$ for scenario A. In such a short time, the spectrum of energy stored in the field that describes both the string and the Nambu-Goldstone radiation cannot change much. Hence, the spectrum of the emitted NG radiation must be $\frac{d E}{d k} \sim \frac{1}{k}$ as was the spectrum of field energy initially stored in the string.

Dabholkar and Quashnock ${ }^{6}$ have carried out a detailed analysis of the collapse of a circular global string loop including radiation reaction effects. They find that in the limit $\ln (R / \delta) \rightarrow \infty$, where $R$ is the radius of the loop, the motion of the string core approaches that predicted by the Nambu-Goto equations. We will see in section IV that this statement is consistent with our computer simulations, although there is a significant quantitative disagreement between their prediction for the motion when $\ell n(R / \delta) \simeq 5$ and our simulation of the same. In addition, Dabholkar and Quashnock conclude that it is always true that in the limit $\ln (L / \delta) \rightarrow \infty$ the motion of the global string core approaches the Nambu-Goto motion, i.e. even for string shapes other than a circular loop. However we find fault with their proof of this assertion. To be specific yet brief, Dabholkar and Quashnock insert 
the general solution of Eq. (1.16a) into the action (1.8) dropping the kinetic term for the Kalb-Ramond field, whereas they should either insert the general solution of Eq. (1.16a) into Eq. (1.16b) or insert it into the total action (1.8) without dropping any terms. Also, Dabholkar and Quashock do not explicitly allow for the possibility of turbulence. As was emphasized above, that assumption can be self-consistent without being correct.

\section{Numerical Techniques}

We wrote a set of programs which iterate in time a classical complex field $\varphi=\varphi_{1}+i \varphi_{2}$ on a box shaped cubic lattice according to the $\frac{\lambda}{4} \varphi^{4}$ Lagrangian:

$$
\begin{aligned}
L= & \sum_{\vec{n}}\left\{\frac{1}{2}\left[\left(\dot{\varphi}_{1}(\vec{n}, t)\right)^{2}+\left(\dot{\varphi}_{2}(\vec{n}, t)\right)^{2}\right]-\sum_{j=1}^{3} \frac{1}{2}\left[\left(\varphi_{1}(\vec{n}+\hat{j}, t)-\varphi_{1}(\vec{n}, t)\right)^{2}\right.\right. \\
& \left.\left.+\left(\varphi_{2}(\vec{n}+\hat{j}, t)-\varphi_{2}(\vec{n}, t)\right)^{2}\right]-\frac{\lambda}{4}\left[\left(\varphi_{1}(\vec{n}, t)\right)^{2}+\left(\varphi_{2}(\vec{n}, t)\right)^{2}-1\right]^{2}\right\},
\end{aligned}
$$

where $\vec{n}$ labels the sites. The sites $\vec{n} \pm \hat{j}(j=1,2,3)$ are the nearest neighbors of $\vec{n}$. The vacuum expectation value $v$, as defined in Eq. (1.2), is set equal to one. It will always be understood that lengths are measured in units of the lattice constant, i.e. the distance between the nearest neighbors. In particular, the core size $\delta=\frac{1}{\sqrt{\lambda}}$ in lattice units. Configurations $\left\{\varphi_{i}(\vec{n}), \dot{\varphi}_{i}(\vec{n})\right\}$ are iterated in time using the algorithm ${ }^{19}$

$$
\begin{aligned}
& \text { 1) } \dot{\varphi}_{i}(\vec{n}, t+\Delta t)=\dot{\varphi}_{i}(\vec{n}, t)+\Delta t \frac{\partial L}{\partial \varphi_{i}(\vec{n})}\left\{\varphi_{j}\left(\vec{n}^{\prime}, t\right)\right\} \quad i=1,2 \text {, all } \vec{n} \notin \Sigma \\
& \text { 2) } \varphi_{i}(\vec{n}, t+\Delta t)=\varphi_{i}(\vec{n}, t)+\Delta t \dot{\varphi}_{i}(\vec{n}, t+\Delta t) \quad i=1,2 \text {, all } \vec{n} \notin \Sigma
\end{aligned}
$$

$\Sigma$ is the set of sites on the boundary of the lattice box. It is important to carry out step 1 of each iteration in its entirety before starting step 2. This algorithm was found to be stable in all cases provided $\Delta t$ was not much larger than 0.5 . The total energy is more and more accurately conserved as $\Delta t$ is made smaller and smaller. Moreover, we found that for $\Delta t \lesssim 0.5$ the energy has no systematic tendency to either increase or decrease.

Similarly, one must adopt a value for $\lambda$ which is not too large. This is because when $\lambda \rightarrow \infty$, we have $\delta \rightarrow 0$ and the "radial" degree of freedom $|\varphi|$ becomes suppressed 
$(|\varphi| \rightarrow 1)$. In that limit, the core of the string is not smooth on the length scale of the lattice constant, and Lorentz invariance is lost. In particular, a straight string moving transversely with initial speed $v_{0}$ will slow down by "grating" on the lattice, whereas in the model of Eq.(1.1) such a string would continue to move with velocity $v_{0}$ forever. We observed that when $\lambda$ is large, the strings slows down by emitting high frequency radiation with wavelength of order the lattice constant. This effect disappears when $\lambda$ is decreased. A sufficiently small value of $\lambda$ is typically 0.5 for simulations of a bent string. For simulations of a collapsing circular loop however, the value of $\lambda$ must be decreased when the initial loop radius $R_{0}$ is increased because the loop of larger initial radius acquires a larger $\gamma=\left(1-\beta^{2}\right)^{-1 / 2}$ value during its collapse and hence its core size become more Lorentz contracted. We found that the $\lambda$ had to be decreased from $\lambda \simeq 0.5$ for $R_{0}<100$ to $\lambda \simeq 0.1$ for $R_{0} \simeq 1000$.

We have used three types of boundary conditions.

a. Dirichelet: $\varphi(\vec{n})$ is constant in time for all $\vec{n} \epsilon \Sigma$.

b. von Neumann: after each iteration (3.2), for all $\vec{n} \epsilon \Sigma$ we set $\varphi(\vec{n})=\varphi\left(\vec{n}^{\prime}\right)$ where $\vec{n}^{\prime}$ is the nearest neighbor site to $\vec{n}$ which does not belong to $\Sigma$.

c. absorptive: after each iteration (3.2), for all $\vec{n} \epsilon \Sigma$ we set $\varphi(\vec{n})=\varphi\left(\vec{n}^{\prime}\right)-h \dot{\varphi}\left(\vec{n}^{\prime}\right)$, where $\vec{n}^{\prime}$ is the nearest neighbor to $\vec{n}$ which does not belong to $\Sigma$. $h$ is a parameter. One recovers Dirichelet boundary conditions in the limit $h \rightarrow \infty$ and von Neumann boundary conditions in the limit $h \rightarrow 0$.

In these two cases (Dirichelet and von Neumann) the boundaries are totally reflecting and hence the total energy in the box is constant. When $h \neq 0$ or $\infty$, the boundaries absorb part of the radiation and energy effectively flows out of the box. 
The total energy inside the box is

$$
\begin{aligned}
E & =\sum_{\vec{n}}\left\{\frac{1}{2}\left[\left(\dot{\varphi}_{1}(\vec{n}, t)\right)^{2}+\left(\dot{\varphi}_{2}(\vec{n}, t)\right)^{2}\right]+\sum_{j=1}^{3} \frac{1}{2}\left[\left(\varphi_{1}(\vec{n}+\hat{j}, t)-\varphi_{1}(\vec{n}, t)\right)^{2}\right.\right. \\
& \left.\left.+\left(\varphi_{2}(\vec{n}+\hat{j}, t)-\varphi_{2}(\vec{n}, t)\right)^{2}\right]+\frac{\lambda}{4}\left[\left(\varphi_{1}(\vec{n}, t)\right)^{2}+\left(\varphi_{2}(\vec{n}, t)\right)^{2}-1\right]^{2}\right\}
\end{aligned}
$$

We will refer to the three successive terms in Eq. (3.3) as $E_{k i n}, E_{\text {grad }}$ and $E_{\text {pot }}$. The potential energy term $E_{\text {pot }}$ is of order $(\ell n L / \delta)^{-1}$ relative to $E_{k i n}$ and $E_{\text {grad }}$. The kinetic plus gradient energy $E_{k i n}+E_{\text {grad }}$ is spectrum analyzed at chosen times during the evolution of the field configuration by Fourier transforming $\dot{\varphi}_{i}(\vec{n})$ for $i=1,2$ and $\varphi_{i}(\vec{n}+\hat{j})-\varphi_{i}(\vec{n})$ for $i=1,2$ and $j=1,2,3$ with respect to $\vec{n}$. This was done using the Cooley-Tukey fast Fourier transform algorithm. We thus calculate $E_{\vec{p}}=E_{\vec{p}}^{g r a d}+E_{\vec{p}}^{k i n}$ for each of the modes $\vec{p}$ in the lattice box. The Fourier transform is defined by

$$
\tilde{f}(\vec{p})=\frac{1}{\sqrt{L_{\mathrm{x}} L_{y} L_{z}}} \sum_{\vec{n}} e^{2 i \pi\left(\frac{p_{x} n_{x}}{L_{x}}+\frac{p_{y} n_{y}}{L_{y}}+\frac{p_{z} n_{z}}{L_{z}}\right)} f(\vec{n})
$$

for $p_{\mathbf{x}}=1 \ldots L_{\mathbf{x}}, p_{y}=1 \ldots L_{y}, p_{z}=1 \ldots L_{z}$. The free oscillation frequency of mode $\vec{p}$ is

$$
k_{\vec{p}}=\sqrt{2\left(1-\cos \frac{2 \pi p_{\mathrm{x}}}{L_{\mathrm{X}}}-\cos \frac{2 \pi p_{y}}{L_{y}}-\cos \frac{2 \pi p_{z}}{L_{z}}\right)} .
$$

We will be particularly interested in the time evolution of

$$
N_{a \mathrm{x}}=\sum_{\vec{p}} \frac{E_{\vec{p}}}{k_{\vec{p}}}
$$

[The manner in which the zero mode $\left(k_{\vec{p}}=0\right)$ is treated when computing $N_{a \mathrm{x}}$ is explained below.] Indeed the continuum version of $N_{a x}$ is

$$
N_{a \mathrm{x}}=\int \frac{d E}{d k} \frac{d k}{k}
$$

After the string has decayed away completely, $N_{a x}$ is the number of axions, i.e. $E / \bar{\omega}$ where $E$ is the total energy and $\bar{\omega}$ is defined in Eq. (1.22). In scenario $A, N_{a x}$ increases by the factor $\ln (L / \delta)$ for that portion of the string energy which is converted into axions. In 
scenario B, on the other hand, $N_{a x}$ is approximately constant. The difference of a factor $\ell n\left(t_{1} / \delta\right) \cong 70$ in the cosmological limits on the axion mass between scenarios $A$ and $B$ precisely follows from this difference in the expected time evolution of $N_{a x}$.

Initial configurations were set up as follows. First, we choose the location of the string core. Next, we adopt a configuration $\varphi^{\prime}(\vec{n})$ which is topologically consistent with that string core position. Next we "relax" this configuration $\varphi^{\prime}(\vec{n})$ using the algorithm

$$
\varphi_{i}(\vec{n}, \tau+\Delta \tau)=\varphi_{i}(\vec{n}, \tau)+\Delta \tau \frac{\partial L}{\partial \varphi_{i}(\vec{n})}\left\{\varphi_{i}\left(\vec{n}^{\prime}, \tau\right)\right\}, \quad i=1,2
$$

with $L$ given by Eq. (3.1), for all lattice sites $\vec{n}$ which are not on the boundary $\Sigma$ and which are more than a distance $\delta$ from the center of the string. After each iteration (3.8) we impose the boundary condition that will be used during the subsequent run. The relaxation algorithm is stable provided $\Delta \tau$ is small enough, say $\Delta \tau=0.1$. It converges to the lowest energy configuration consistent with the boundary conditions and with the position of the string core. For each of the simulations described below the initial $\dot{\varphi}_{i}(\vec{n})$ were set equal to zero. In each case we verified that the initial configuration was sufficiently relaxed by checking that further relaxation did not significantly alter the results of the subsequent run.

\section{Simulations of Collapsing Circular Loops}

The static axion field carried by a loop located at $\vec{x}_{0}(s)$, where $s$ is a parameter labeling the points along the loop, is

$$
a(\overrightarrow{\mathrm{x}})=\frac{v}{2} \Omega(\overrightarrow{\mathrm{x}})
$$

where $\Omega(\vec{x})$ is the solid angle subtended by the loop when viewed from location $\vec{x}$. Indeed, $\nabla^{2} \Omega(\vec{x})=0$ for $\vec{x} \notin\left\{\vec{x}_{0}(s)\right.$, all $\left.s\right\}$ and the change in $\alpha(\vec{x})=\frac{a(\vec{x})}{v}=\frac{1}{2} \Omega(\vec{x})$ upon circling the string core anywhere is $2 \pi$. One can use (4.1) to set up an initial configuration corresponding to an arbitrary loop location, by setting $\varphi(\overrightarrow{\mathrm{x}})=v e^{i \Omega(\overrightarrow{\mathrm{x}}) / 2}$ for all $\overrightarrow{\mathrm{x}}$ far away from the 
string core and $\varphi(\vec{x})=v f(r) e^{i \Omega(\vec{x}) / 2}$ for $\overrightarrow{\mathrm{x}}$ close to the string core, where $f$ is the function defined by Eqs. (1.5) and (1.6) and $r$ is the distance to the string core center. For our circular string loop simulations, we used this method to set up the initial pre-relaxation configuration $\varphi^{\prime}(\vec{n})$. This $\varphi^{\prime}(\vec{n})$ configuration was then thoroughly relaxed before the run actually began, as described above.

For all our circular loop simulations, the lattice box is a cube of volume $L^{3}$. The boundary conditions on all surfaces are von Neumann type. We found that finite volume effects are negligible when the diameter of the initial string loop is smaller than 0.6 times the sice $L$ of the box.

We have carried out 2-dimensional and 3-dimensional simulations of the collapse of circular string loops to determine the time dependence of the radius $R(t)$. The 2-dim. simulations exploit the circular symmetry of the problem. The computation is carried out on a 2-dim. lattice which covers the piece of plane $0 \leq z \leq L / 2,0 \leq \rho \leq L / 2$ in cylindrical coordinates in which the loop is in the $z=0$ plane. Because of $z \rightarrow-z$ reflection symmetry, one does not need to cover the $-L / 2 \leq z \leq 0$ area. Our largest simulations of this type had $L / 2=1500$ and initial radius $R_{0}=900$. The computation for the 3 -dim. simulations are carried out on a lattice which covers the volume $0 \leq \mathrm{x}, y, z \leq L / 2$ in cartesian coordinates in which the loop is in the $z=0$ plane. Here, we exploit the symmetries $x \rightarrow-x, y \rightarrow-y$ and $z \rightarrow-z$. Our largest 3-dim. simulations had $L / 2=150$ and $R_{0}=90$. It might at first appear a waste of time to do the 3-dim. simulations, but that is not necessarily true. Indeed, small deviations from circular symmetry may grow so fast that the assumption of circular symmetry is unrealistic. One way to test for this is to compare the 2-dim. simulations with the 3-dim. ones.

Fig. I shows $R(t)$ for our largest 3-dim. simulation, whereas Fig. II shows $R(t)$ for our largest 2-dim. simulation. By definition, $R$ is the radius of the string core center, i.e. where $\varphi(\vec{x})$ vanishes. $R(t)$ is shown by the solid line in Figs. I and II. The dashed line 
shows the time dependence of the "center of energy" radius $R_{E}(t$ defined by 8

$$
R_{E}=\frac{\int d \rho d z \rho^{2} \mathcal{H}(z, \rho)}{\int d \rho d z \rho \mathcal{H}(z, \rho)}
$$

where $\mathcal{H}(z, \rho)$ is the energy density. Approximately three years ago, Vachaspati ${ }^{8}$ carried out 2-dim. (i.e. assuming circular symmetry) simulations of the collapse of circular global string loops and noticed several interesting features which have also manifested themselves in our simulations. The size of his lattice was approximately $180 \times 180$. He observed among other things that the position of the string core $R(t)$ does not coincide with the string center of energy $R_{E}(t)$. This is also evident in Figs. I and II. We find however that the relative difference between $R_{E}(t)$ and $R(t)$ decreases as $\log \left(R_{0} / \delta\right)$ is increased.

We made many comparisons between 3-dim. simulations and 2-dim. ones with the same parameters $\left(R_{0}, \lambda\right)$. In each case, we found that the 2-dim. and 3-dim. simulations produced $R(t)$ and $R_{E}(t)$ curves which are almost exactly the same. In this regard, at least, the assumption of circular symmetry appears to be realistic.

The simulations shown in Figs I and II cannot be trusted beyond the point where the collapse has occurred, because the computer code cannot adequately track the large amount of energy which is localized on a small number of lattice sites near the time of collapse. Nonetheless, Fig. I suggests that there is a small rebound, i.e. after the collapse, the loop re-expands albeit to a much smaller radius than $R_{0}$ and in a rather irregular fashion. To study this rebound, we ran several simulations with small values of $\Delta t$ and $\lambda$. The rebound increased in size up to a radius equal to about $15 \%$ of $R_{0}$. Ii continued to be very chaotic however and did not increase any further in size with decreasing $\Delta t$ and $\lambda$.

We carried out many simulations of the collapse of circular loops for different values of $R_{0}, \lambda$ and $\Delta t$. Fig. III shows $T / R_{0}$ vs $\ln \left(R_{0} / \delta\right)$ where $T$ is the collapse time, i.e. the amount of time it takes $R(t)$ to change from $R_{0}$ to zero. The solid triangle data poins are the result of 2-dim. simulations whereas the empty triangle data points are the result of 
3-dim. simulations. For each data point, it was verified that $\lambda$ and $\Delta t$ were sufficiently small that the continuous space and time limits had been reached (cfr. section III). The size of the triangles express our rough estimate of the remaining uncertainties. The solid line at $T / R_{0}=\pi / 2$ is the Nambu-Goto limit. Indeed, a circular string loop obeying the Nambu-Goto equations of motion collapses according to $R(t)=R_{0} \cos \left(t / R_{0}\right)$. This motion can be obtained by solving

$$
E=2 \pi \tau R\left(1-\dot{R}^{2}\right)^{-1 / 2}
$$

for $R(t)$, with $E$ and $\tau$ constant. $E$ and $\tau$ are respectively the total energy and tension of the Nambu-Goto string. One might wonder what the assumption that the energy of the string is conserved by itself would imply for the motion of a circular global string loop. Of course, the motion obtained in this manner should not be expected to be necessarily correct since the global string can emit NG radiation and thus its energy is not necessarily conserved. Only the total (string + radiation) energy is guaranteed to be conserved. At any rate, the analog of Eq. (4.3) for a global string is

$$
E=2 \pi^{2} v^{2} R \ell n(R / \delta)\left(1-\dot{R}^{2}\right)^{-1 / 2}
$$

obtained by setting $\tau=\pi v^{2} \ln (R / \delta)$ in Eq.(4.3). Eq.(4.4) takes account not only of the string tension force $\pi v^{2} \ln (R / \delta)$ but also of the force $O\left(\pi v^{2}\right)$ mediated by the NG field between different parts of the string. Eq. (4.4) with $E=$ constant yields

$$
T / R_{0}=\int_{0}^{1} \frac{d \mathrm{x}}{\left[1-\mathrm{x}^{2}\left(1+\frac{\ln \mathrm{x}}{\ln R_{0} / \delta}\right)^{2}\right]^{1 / 2}}
$$

which is represented by the dashed line in Fig. III. In the paper by Dabholkar and Quashnock ${ }^{6}$, their expected time evolution for $R(t)$ is displayed in a figure for $\ell n\left(R_{0} / \delta\right)=$ 5. The corresponding prediction for $T / R_{0}$ very closely agrees with Eq. (4.5). Our computer simulations on the other hand clearly disagree with Eq. (4.5). They are consistent with the statement that $T / R_{0}$ approaches the Nambu-Goto value of $\pi / 2$ in the $\ln \left(R_{0} / \delta\right) \rightarrow \infty$ 
limit. However, over the range of $\ln \left(R_{0} / \delta\right)$ values investigated $\left(2 \lesssim \ln \left(R_{0} / \delta\right) \lesssim 6\right)$, the deviation of $T / R_{0}$ from $\pi / 2$ is roughly twice as large as that predicted by Eq. (4.5).

Finally, we turn to our cesults on the evolution of the energy spectrum of the field $\varphi$ which describes both the string and the NG radiation. As explained at the end of section III, the kinetic and gradient field energy in the whole box is spectrum analyzed at chosen times during and after the collapse of the circular loop. The lattice has von Neumann type boundary conditions. Hence the total energy inside the box is conserved. Spectrum analysis was only carried out in conjunction with simulations which are 3-dimensional and which do not exploit the $x \rightarrow-x, y \rightarrow-y$ and $z \rightarrow-z$ reflection symmetries. The largest simulations we $\operatorname{ran}$ had $R_{0}=40$ and $L=128$. Fig. IV shows the spectrum $\frac{d E}{d \ell n k}$, disp'ayed in $P n k$ bins of width $\Delta l n k=1$, before the collapse of a loop of radius $R_{0}=40$ has started (white histogram) and after the collapse has been completed (black histogram). The energy in the zero mode $\vec{p}=0$ [see Eq. (3.5)] is displayed in the bin labeled $\ell n k=-\infty$. The bin which contains the lowest non-zero mode has arbitrarily been called $\ell n k=3$. The initial spectrum is not very flat because $\ln \left(R_{0} / \delta\right)$ is not large. For larger values of $\ln \left(R_{0} / \delta\right)$ a flat plateatu would appear in the middle region of the spectrum. This is discussed in greater detail in the next section. At any rate, Fig. IV shows that the spectrum hardens very markedly during the collapse. This hardening occurred in each of our simulations with spectrum analysis of the collapse of a circular loop.

Fig. $V$ shows the time evolution of $N_{a x}$ during the collapse of circular string loops of initial radius $R_{0}=40$ (solid line) and $R_{0}=20$ (dashed line). $N_{a x}$ was defined in Eqs. (3.6) and (3.7). The energy in the zero mode $(\vec{p}=0)$ is not included in the computation of $N_{a x}$ for Fig. V. To make the comparison between the $R_{0}=40$ and $R_{0}=20$ curves more straightforward, $N_{a \mathrm{x}}$ was normalized to its initial value $N_{a \mathrm{x}_{0}}$ and time was normalized to the collapse time $T$. The figure shows that:

1) $N_{a x}$ decreases during the collapse. (This is of course consistent with the hardening of 
the spectrum shown in Fig. IV.)

2) there is little change in the evolution of $N_{a x}$ when $R_{0}$ is changed from 20 to 40 , which corresponds to an increase in $\ell n\left(R_{0} / \delta\right)$ of $\ell n 2=0.69$.

Recall that in scenario $\mathrm{A}, N_{a \mathrm{x}}$ increases by the factor $\ln (L / \delta)$ when a string loop decays into NG radiation. However that expectation may be inapplicable to the decay of a circular loop because a circular loop can decay to a point in one go. In scenario A, an arbitrary non-circular loop is expected to oscillate. Let us visualize such an oscillation as changing the initial length $L$ of the loop down to $L / 2$, for example, then back to $L$ and so forth. Presumably, during the $I_{1} \rightarrow L_{/}^{\prime} 2$ half-period, $N_{a x}$ will decrease as in the first third of Fig. $\mathrm{V}$ because the shape of the loop is most likely irrelevant to this effect. After all, during the contraction half of the oscillation one should expect all the modes to get blue-shifted. In scenario $A$ then it must be so that during the expansion $(L / 2 \rightarrow L)$ half-period, $N_{a x}$ increases back to roughly twice its original value. After $\ln (L / \delta)$ oscillations the overall increase in $N_{a \mathrm{x}}$ would be from $N_{a x_{0}}$ to $\ln (L / \delta) N_{a x_{0}}$. To see whether this effect exists is part of our motivation to carry out the simulations of a bent string described in the next section. In these simulations, the boundary conditions keep the bent string in existence. The bent string simulations thus provide the best possible environment for the validation of scenario A.

We did carry out a few simulations of non-circular loops. In these simulations, the loops collapsed at once, without any oscillation. The spectrum evolved in much the same way as for the collapse of circular loops. Such an outcome is what one would expect on the basis of the bent string simulations described in the next section. 


\section{Simulations of a Bent String}

We use a rectangular box-shaped lattice of size $L_{x} L_{y} L_{z}$. The string is parallel to the $\hat{z}$-direction when straight. Dirichelet-type boundary conditions are imposed on the top and bottom plates $\left(n_{z}=0\right.$ and $\left.n_{z}=L_{z}\right)$ by holding $\varphi(\vec{n})$ fixed there at its values for a straight string. The boundary conditions on the side plates $\left(n_{x}=0, L_{x}\right.$ and $\left.n_{y}=0, L_{y}\right)$ are von Neumann type unless stated otherwise. The initial configuration is one in which the core position is displaced by the amount $A_{0} \sin \left(\frac{2 \pi n_{n}}{L_{z}}\right)$ in the $\hat{x}$-direction. The problem has a double reflection symmetry, one along the $\hat{z}$-axis and one along the $\hat{y}$-axis. When we simulate the motion without spectrum analysis, we exploit this double reflection symmetry to carry out the computation only on the $\left\{0 \leq x \leq L_{x}, 0 \leq y \leq L_{y} / 2,0 \leq z \leq L_{z} / 4\right\}$ part of the lattice box. This allows us to reach somewhat larger values of $\ln (L / \delta)$ in those simulations. When we simulate the motıon with spectrum analysis, however, we carry out the computation over the whole lattice box. The initial pre-relaxation configuration which we use is

$$
\varphi^{\prime}(\vec{n})=\varphi_{0}\left(\vec{n}-\hat{x} A_{0} \sin \frac{2 \pi n_{z}}{L_{z}}\right)
$$

where $\varphi_{0}(\vec{n})$ is the configuration of the straight string given in Eq.(1.3). This $\varphi^{\prime}(\vec{n})$ configuration is then thoroughly relaxed, as described in section III, to obtain the initial configuration with which the simulation actually begins.

Fig. VI shows the time evolution of the amplitude $A(t)$ for two runs with different initial amplitudes, but for which everything else is the same. In both cases, the box size is $L_{x}\left(L_{y} / 2\right)\left(L_{z} / 4\right)=240 \times 120 \times 18.5$. Qualitatively, the motion of a bent string in our simulations can be described as follows. If the amplitude $A_{0}$ is larger than a critical amplitude $A_{\text {crit }}$ of order $10 \%$ of the wavelength $\Lambda$ of the bent, the string will damp strongly so that after one or two oscillations, $A(t)$ is less than $A_{\text {crit }}$. Once $A(t)$ is less than $A_{\text {crit }}$, the damping is much less. If $A_{0}$ is increased to several times $A_{\text {crit }}$, it still remains qualitatively true that $A(t)$ decreases to below $A_{\text {crit }}$ in one or two oscillations. Fig.VI illustrates this. 
After the first few oscillations shown in Fig. VI the motion becomes less regular. This is because the radiation which was emitted by the string during its early period of strong damping has bounced back from the sides of the box, has returned in part to the location of the string and is now driving it. Aside from these irregularities, the amplitude has a long term time dependence (not shown in Fig. VI) which is akin to beating. Most likely this behaviour is in fact due to the beating of the string against the several modes in the box which have the same oscillation frequency as the string.

We also carried out a few simulations with absorptive boundary conditions on the side plates. As previously, for large enough $A_{0}, A(t)$ decreases in one or two oscillations to a value below $A_{\text {crit }} \simeq 0.1 \Lambda$. After that, it decays more slowly. Instead of beating, it now decays uniformly to near zero. However, this latter decay rate depends on the absorption coefficient of the side plates, i.e. on the value of $h$ used in imposing the boundary condition there.

In the simulations of a bent string, the infra-red cutoff $L$ is given by the wavelength $\Lambda$ because the field $\varphi$ at points much more than a distance $\Lambda$ away from the string core does not participate in the oscillations whereas the field at points within a distance of order $\Lambda$ from the string core does participate in the oscillations. To investigate the dependence of the motion upon $\ln (\Lambda / \delta)$, we made several series of runs in which $L_{x}, L_{y}, L_{z}$ and $A_{0}$ are all changed by a common factor whereas the core size $\delta=\frac{1}{\sqrt{\lambda}}$ is held fixed. Fig. VII shows a series of three such runs in which $\Lambda$ is increased by successive factors of approximately 1.8. From one run to the next, $\ln (\Lambda / \delta)$ is thus increased by $\ln (1.8) \simeq 0.6$. To make the comparison between the three runs more straightforward, the time is normalized to the wavelength $\Lambda$ whereas the amplitude is normalized to its initial value $A_{0}$. In each of the three runs, $A_{0} \simeq 0.2 \Lambda$. Fig.VII shows that when $\ln (\Lambda / \delta)$ is increased, the critical amplitude $A_{\text {crit }}$ increases also, i.e. the string is able to sustain long term oscillations of intreasing añplitude. Oñ the othes handu, the second derivative of $A_{\text {crii }}$ with respect to 
$\ell n(\Lambda / \delta)$ is negative. It is possible that for larger $\ln (\Lambda / \delta), A_{c r i t}$ stops increasing with $\ln (\Lambda / \delta)$. However, our simulations did not have sufficiently large $\ln (\Lambda / \delta)$ to settle the issue.

In regard to the motion of bent global strings, our results are consistent with the simulations of Davis and Shellard ${ }^{5}$, although their description of the motion has a different qualitative emphasis.

Next, we turn to the spectrum analysis results for the bent string simulations. Fig. VIII shows the spectrum of a straight string with core size $\delta=\frac{1}{\sqrt{\lambda}}=\frac{1}{\sqrt{0.5}}$. in a box of transverse dimensions $L_{\mathrm{x}}=L_{y}=4096$. Because this configuration is z-independent, we could set $L_{z}=1$ and thus reach relatively large values of $L_{\mathrm{x}}$ and $L_{y}$ and hence of $\ln (L / \delta)$. The figure shows a clean flat plateau between $\ell n(k)=3.0$ and $\ell n(k)=5.5$. Note that the plateau value of $\frac{d E}{d \ell n k}$ is consistent with $\pi=3.14 \ldots$ expected from Eq. (1.7). The with of the plateau increases linearly with $\ln (L / \delta)$. When the string is bent, the spectrum analysis must be carried out in 3 dimensions, and the values of $\ln (L / \delta)$ are much smaller. The width of the central plateau is correspondingly less. This is evident in Fig. IX which shows the spectra of three static $[\dot{\varphi}(\vec{n})=0]$ string configurations of amplitudes $\mathrm{A}=26$, 13 and 0 in a box of size $L_{\mathbf{x}}=L_{y}=L_{z}=128$. The difference between the $\mathrm{A}=26$ and $A=0$ spectra is the energy available for release into $N G$ radiation when a bent string of initial amplitude $A_{0}=26$ relaxes. For $\Lambda=128$, the critical amplitude is of order 13. Fig. IX shows that a bent string with amplitude of order $A_{\text {crit }}$ has very little energy available for release into NG radiation.

We ran many bent string simulations with spectrum analysis at various chosen times while the string is relaxing. Table I shows a typical run. The box size was $(128)^{3}$. The initial amplitude was $A_{0}=20$. The spectrum of the kinetic plus gradient fieli energy is shown at six different times binned in bins of width $\Delta l n k=\frac{1}{3}$. The energy in the zero mode $(\mathrm{k}=0)$ is shown in the bin labeled $-\infty$. The bin containing the lowest non-zero 
Table I.

\begin{tabular}{|c|c|c|c|c|c|c|c|c|}
\hline & bin \# & \# modes & $t=0$ & 200 & 400 & 600 & 800 & 1000 \\
\hline \multirow[t]{2}{*}{$(0,0,0)$} & $-\infty$ & 1 & 390 & 357 & 367 & 371 & 350 & 378 \\
\hline & 5 & 0 & 0 & 0 & 0 & 0 & 0 & 0 \\
\hline$(1,0,0) \ldots$ & 6 & 6 & 279 & 337 & 329 & 326 & 368 & 326 \\
\hline$(1,1,0) \ldots$ & 7 & 12 & 215 & 170 & 183 & 195 & 152 & 205 \\
\hline \multirow[t]{13}{*}{$(2,0,0) \ldots$} & 8 & 38 & 165 & 202 & 195 & 198 & 223 & 208 \\
\hline & 9 & 90 & 163 & 161 & 167 & 160 & 164 & 154 \\
\hline & 10 & 242 & 140 & 167 & 154 & 153 & 145 & 145 \\
\hline & 11 & 656 & 143 & 138 & 139 & 132 & 136 & 131 \\
\hline & 12 & 1900 & 128 & 115 & 113 & 119 & 116 & 111 \\
\hline & 13 & 5224 & 96 & 87 & 86 & 93 & 94 & 91 \\
\hline & 14 & 14526 & 60 & 50 & 48 & 55 & 54 & 52 \\
\hline & 15 & 42740 & 30 & 22 & 21 & 26 & 25 & 23 \\
\hline & 16 & 138924 & 10 & 9 & 8 & 12 & 10 & 7 \\
\hline & 17 & 632559 & 2.1 & 15 & 12 & 23 & 15 & 10 \\
\hline & 18 & 1170893 & 0.8 & 6.7 & 5 & 12 & 5 & 3.3 \\
\hline & 19 & 89341 & 0.13 & 0.23 & 0.24 & 0.23 & 0.23 & 0.24 \\
\hline & 20 & 0 & 0 & 0 & 0 & 0 & 0 & 0 \\
\hline Total & & 2097152 & 1822 & 1837 & 1829 & 1873 & 1854 & 1845 \\
\hline $\begin{array}{l}\text { Potential } \\
\text { enerey }\end{array}$ & & & 196 & 207 & 204 & 184 & 182 & 182 \\
\hline $\begin{array}{l}\text { Total } \\
\text { energy }\end{array}$ & & & 2018 & 2044 & 2034 & 2057 & 2035 & 2027 \\
\hline
\end{tabular}


modes has arbitrarily been called \#6. The total energy, shown in the bottom row, is conserved within $2 \%$. The energy in the six modes $[(1,0,0),(0,1,0) \ldots]$ with frequency nearly equal to $\omega_{0} \equiv \frac{2 \pi}{\Lambda}$ (strictly equal in the continuum limit) is shown in bin \#6. $\omega_{0}$ is also the oscillation frequency of the string in the limit of small amplicudes. Similarly, bin \#7 contains the twelve modes $[(1,1,0) \ldots]$ of frequency $\sqrt{2} \omega_{0}$; bin $\# 8$ contains the six modes $[(2,0,0) \ldots]$ of frequency $2 \omega_{0}$ and thirty-two other modes $[(2,1,0) \ldots]$ with frequency near $2 \omega_{0}$; and so on. Tabie I shows that the energy in the fundamental (bin \#6) and first harmonic (bin \#8) increases while the string relaxes. On the other hand, the energy in the zero mode (bin $-\infty$ ) and ir. bin \#7 decreases. The overall resuit is a relatively small increase in the low wavevector bins. There is also a small increase in the highest wavevector bins and a depletion in the intermediate wavevector region. Correspondingly, $N_{a x}$ changes very little throughout the run. We have observed this general behaviour in all our bent string simulations. Figs. X and XI show the time evolution of $N_{a \mathrm{X}}$ in four representative cases. For the purpose of these figures, in the computation of $N_{a x}$ [see Eq. (3.6)] the energy in the zero mode $(k=0)$ is treated as if it were in the lowest non-zero mode. The figures show that $N_{a x}$ does not have any clear tendency to either increase or decrease. In scenario $\mathrm{A}$, on the other hand, the difference in string energy between the initial and final states of the string is converted into NG radiation of frequency $\omega_{0}$. This statement is clearly incompatible with our simulations. To give a specific example, in the run with initial amplitude $A_{0}=30$ on the $(128)^{3}$ lattice shown in Fig. XI, $N_{a x}$ would have increased from its initial value of $2.410^{4}$ to a value of order $2.9510^{4}$ in about two oscillation periods, i.e. after a time of order $2 \Lambda \simeq 260$. This is strongly inconsistent with the similation.

Thus far none of our figures show a pure radiation spectrum, i.e. the spectrum of NG radiation emitted by the string separated from the spectrum of field energy of the string itself. One may fear that there is something misleading about looking only at the total 
(radiation + string field) energy spectrum. Fig. XII is intended to dispell such doubts It shows results from a simulation in a box of size $(64)^{2} \times 192$ which can be thought of as three cubes of volume $(64)^{3}$ side by side. The string is in the right hand side cube. Its initial anplitude is $A_{0}=15$. The white histogram in Fig. XII shows the difference in the energy spectra between the initial $\left(A_{0}=15\right)$ and straight $\left(A_{0}=0\right)$ configurations of that string. The field energy in the left hand side cube is spectrum analyzed at many regular time intervals after the string has been released. Of course, the energy inside the LHS box is not conserved. At first, the box is almost empty; it contains only a small amount ci static field energy. After a time interval sufficient for the emitted radiation to travel from the string to the LHS box, the energy in the LHS box suddenly increases by more than a factor of ten. After that time, almost all the energy inside the box is radiation. The spectrum changes somewhat from one measurement to the next. We took $\sim 80$ spectra and averaged them. The average spectrum is the black histogram in Fig. XII. The figure clearly speaks its support for scenario B.

In regard to the spectrum analysis of $N G$ radiation emitted by a relaxing/oscillating bent string, our results are in disagreement with those of Davis and Shellard ${ }^{5}$. Using a technique different from ours, they obtained a spectrum which is very narrowly peaked at a frequency equal to twice the oscillation frequency of the string.

\section{Summary and Conclusions}

Two contrasting scenarios have been put forth in the literature for the motion and decay of global strings. In scenario A, the energy spectrum of the field that describes both the global string and the accompanying Nambu-Goidstone (NG) boson is modified from an initially flat spectrum - i.e. $\frac{d E}{d \ell n k} \simeq$ constant with a low wavevector cutoff of order $1 / L$ and a high wavevector cutoff of order $1 / \delta$ where $\delta$ is the core size of the global string and $L$ is the length scale over which it is bent - to one which is peaked at the wavevector $1 / L$. 
As a result, the quantity

$$
N_{a x}=\int \frac{d E}{d k} \frac{d k}{k}
$$

increases in scenario $\mathrm{A}$ by the factor $\ell n(L / \delta)$ while the global string decays into NG radiation. In scenario $B$, on the other hand, the spectrum does not change appreciably while the string decays. As a result, $N_{a x}$ remains roughly constant in scenario B and, specifically, does not increase by the factor $\ell n(L / \delta)$.

The problem of the motion and decay of global strings is relevant to the determination of the cosmological constraint on the axion mass in the case where there is no inflation after the phase transition in which the $U_{P Q}(1)$ symmetry of Peccei and Quinn get spontaneously broken. Because the number of cosmic axions produced by cosmic axion strings is larger by the factor $\ln (L / \delta) \simeq \ln \left(t_{Q C D} f_{a}\right) \simeq 70$ in scenario $\mathrm{A}$ than it is in scenario $\mathrm{B}$, the constraint on the axion mass is correspondingly more severe. The problem of the motion and decay of global strings is also relevant to a recent interpretation of certain cosmic ray and radioastronomy observations as due to the conversion of Nambu-Goldstone bosons to photons in astrophysical fields. Crucial to this interpretation is the assumption that the spectrum of NG radiation from cosmic global strings is $\frac{d E}{d k} \sim \frac{1}{k}$ as in scenario $\mathrm{B}$.

If a global string initially bent over a length scale $L$ is perfectly smooth and remains perfectly smooth during its subsequent motion - i.e. if there are no short wavelength wiggles or other rapidly oscillating structures on the string -, then the NG radiation emitted by the string will be peaked at the oscillation frequency, of order $1 / L$, of the string and one of the necessary conditions for scenario A will be satified. However, many strongly coupled non-linear systems exhibit turbulence. If small wiggles are present on the string either as an initial condition or as spontaneously generated turbulence, then scenario A will fail provided the wiggles are not too small. For a global string to radiate away its excess energy at all frequencies at once, it is sufficient it has wiggles with amplitude $d_{\omega} \sim \frac{1}{\omega \sqrt{\omega L}}$ for all $\omega$. 'This vanishes in the $L \rightarrow$ limit, indicating that very small turbulence on the 
string is sufficient to shake off the energy stored in wavevectors $k>>\frac{1}{L}$. For example, for $\omega=1 G H_{z}$ and $L=t_{0}=10^{10}$ years, the amplitude of the wiggles need not be larger than $10^{-13} \mathrm{~cm}$. Scenario A may also fail to be correct because of self-intersections of the global string with reconnection of the intersecting pieces as explained in section II. Generally speaking, it is easy to find processes that harden the initial $\frac{d E}{d k} \sim \frac{1}{k}$ spectrum of a global string, instead of softening it as in scenario $A$, because the mode density of the field that describes both the global string and the NG radiation goes like $\frac{d n}{d k} \sim k^{2}$ and the overall effect of a process is usual, in the direction of spreading the energy equally over all modes. Such processes may allow the energy stored in the initial non-straight string configuration to be released on all wavevector scales more or less equally. If they do occur, one will obtain scenario $B$ for the decay rate and radiation spectrum of global strings.

Dabholkar and Quashnock ${ }^{6}$ (DQ) have argued in favor of scenario $A$ on the basis of analytical calculations intended to take account of the back-reaction of the emitted radiation upon the string core. DQ use approach to Nambu-Goto motion in the $L \rightarrow \infty$ limit as a criterion to distinguish between scenarios A and B. But it is unclear precisely how this criterion should be applied. Indeed, we have just seen that turbulence of frequency $\omega$ and amplitude $\frac{1}{\omega \sqrt{\omega L}}$ makes all the difference between the two scenarios. Yet that difference vanishes in the $L \rightarrow \infty$ limit for fixed $\omega$. The first part of the DQ paper is supposed to show that in the $L \rightarrow \infty$ limit, the global string always satisfies the NambuGoto equations of motion. Apart from our previous comment, we disagree with their proof on the technical grounds explained at the end of section II. Finally, the motion obtained by $D Q$ for a collapsing circular loop when $\log (L / \delta) \simeq 5$ disagrees with our computer simulation of same.

Davis and Shellard ${ }^{5}$ (DS) have argued in favor of scenario A on the basis of computer simulat ins of oscillating/relaxating bent strings. The motion of the string is obtained by integrating the classical $\lambda \varphi^{4}$ equations of motion, as in our simulations. The motion they 
obtain is consistent with our results although their description, of it has a very different qualitative emphasis. The techniques they use for the spectrum analysis of the NG radiation are different from ours, and the results are different as well. DS find that the spectrum of NG radiation is peaked at twice the oscillation frequency of the string. This is strongly inconsistent with our results for the NG radiation spectrum, as shown for example by Fig. XII.

The results from our own computer simulations may be summarized as follows.

For collapsing circular loops $\left(2 \lesssim \ln \left(R_{0} / \delta\right) \lesssim 4\right.$ in the 3 dim. simulations):

- the motion approaches that predicted by the Nambu-Goto equations in the large $\ell n\left(R_{0} / \delta\right)$ limit (see Fig. III). The motion is the same whether simulated in $2 \mathrm{dim}$. (exploiting the circular symmetry) or in $3 \mathrm{dim}$.

- there is a marked hardenirg of the spectrum during the collapse and an overall decrease of $N_{a x}$ by about $25 \%$ (see Figs. IV and V). No appreciable change in this phenomenon is seen when $\ln \left(R_{0} / \delta\right)$ is increased.

For bent strings $(2.5 \lesssim \ell n(\Lambda / \delta) \lesssim 4.5)$ :

- the motion is strongly dariped for uscillations of amplitude larger than a critical amplitude $A_{c}$ of order 0.1 times the wavelength of the bent (see Fig. VI). Over the range of $\ln (\Lambda / \delta)$ values investigated, the first derivative of $A_{c}$ with respect to $\ln (\Lambda / \delta)$ is positive whereas the second derivative is negative (see Fig. VII).

- the spectrum changes little during the motion of the bent string. Correspondingly, $N_{a x}$ is very nearly constant (see Figs. $\mathrm{X}$ and XI). No appreciable change in the behaviour of $N_{a x}$ occurs when $\ln (\Lambda / \delta)$ is increased. The spectrum of NG radiation is similar to the spectrum of field energy stored in the string (Fig. XII).

Over the range of $\ln (L / \delta)$ values that can be investigated, our simulations are consistent with scenario $B$. They are inconsistent with scenario A with regard to the spectrum of 
emitted NG radiation. Of course, the $\ell n(L / \delta)$ values of cosmological interest are much larger $(\sim 100)$ than the values that were accessible by our computer simulations. The behaviour of global strings can certainly change when $\ell n(L / \delta)$ is increased. We argued however that there are general circumstances favoring scenario B for all $\ln (L / \delta)>>1$.

\section{Acknowledgements:}

We are very grateful to D. Harari and A. Polychronakos for the numerous conversations during which they gave us the benefit of their interpretations and criticism. We would also like to thank J. Quashnock, A. Dabholkar, T. W. B. Kibble, P. Shellard, R. Holman, J. Ellis, M. Turner, J. Harvey, N. Turok and D. Bennett for stimulating discussions. One of us (P.S.) would like to thank the Aspen Center for Physics for its hospitality while he was working on this project.

This work was supported in part by the U.S. Department of Energy under contract no. DE-FG05-86ER40272, and by the University of Florida and the IBM Corp. through their Research Computing Initiative at the Northeast Regional Data Center.

\section{References}

1. T. W. B. Kibble, J. Phys. $\underline{\text { A9 }}$ (1976) 1387; Phys. Rep. $\underline{67}$ (1980) 183.

2. For a review on Cosmic Strings, see: A. Vilerkin, Phys. Rep. 121 (1985) 264.

3. R. Davis, Phys. Rev. D32 (1985) 3172, and Phys. Lett. 180B (1986) 225.

4. A. Vilenkin and T. Vachaspati, Phys. Rev. D35 (1987) 1138.

5. R. L. Davis and E. P. S. Shellard, Nucl. Phys. B324 (1989) 167.

6. A. Dabholkar and J. M. Quashnock, Nucl. Phys. B333 (1990) 815.

7. D. Harari and P. Sikivie, Phys. Lett. B195 (1987) 361. 
8. T. Vachaspati, "Global String Dynamics", Cambridge University preprint, 1988 (unpublished).

9. B.-A. Gradwohl, G. Kälbermann, T. Piran and E. Bertschinger, "Global Strings and Superfluid Vortices: Analogies and Differences" Hebrew University preprint HUT$140 / 89$ (December 89).

10. M. Kalb and P. Ramond, Phys. Rev. D9 (1974) 2273; F. Lund and T. Regge, Phys. Rev. D14 (1976) 1524.

11. E. Witten, Phys. Lett. 158B (1985) 243.

12. P. Sikivie, Phys. Rev. Lett. 48 (1982) 1156.

13. A. Vilenkin and A. E. Everett, Phys. Rev. Lett. 48 (1982) 1867; T. W. Kibble, G. Lazarides and Q. Shafi, Phys. Rev. D26 (1982) 435. P. Sikivie, "Axions in Cosmology" in Where are the Elementary Particles, Proc. 14th Summer School on Particle Physics (Gif-sur-Yvette, 1982), eds. P. Fayet et al.

14. L. Abbott and P. Sikivie, Phys. Lett. 120B (1983) 133; J. Preskill, M. Wise and F. Wilczek, Phys. Lett. 120B (1983) 127; M. Dine and W. Fischler, Phys. Lett. 120B (1983) 137.

15. G. Raffelt and D. Seckel, Phys. Rev. Lett. $\underline{60}$ (1988) 1793; M. S. Turner, Phys. Rev. Lett. $\underline{60}$ (1988) 1797; R. Mayle et al., Phys. Lett. 203B (1988) 199; T. Hatsuda and M. Yoshimura, Phys. Lett. 203B (1988) 469; A. Burrows, M. S. Turner and R. P. Brinkman, Phys. Rev. D39 (1989) 1020; N. Iwamoto, Phys. Rev. D39 (1989) 2120; A. Burrows, M. T. Russell, and M. S. Turner, "Axions and SN1987a", to be published.

16. P. Sikivie, Phys. Rev. Lett. 61 (1988) 783; and in the Proceedings of the Workshop on Cosmic Axions, edited by C. Jones and A. Melissinos, World Scientific, 199C, p. 233.

17. P. Sikivie, Phys. Rev. Lett. $\underline{51}$ (1983) 1415. 
18. D. Bennett and A. Bouchet, Phys. Rev. Lett. 60 (1988) 257 and Phys. Rev. Lett. $\underline{63}$ (1989) 1334; B. Allen and E. P. S. Shellard, MIT preprint (1989). For earlier work see: A. Albrecht and N. Turok, Phys. Rev. Lett. $\underline{54}$ (1985) 1868 and Phys. Rev. D40 (1989) 973.

19. D. Harari and A. Polychronakos, private communication. 


\section{Figure Captions:}

Fig. I: Time evolution of the radius $R$ (solid line) and the "center of energy" radius $R_{E}$ (dashed line) during the collapse of the circular global string loop in our largest 3-dim. simulation. $R_{0}=90, L / 2=150$ and $\lambda=0.5$.

Fig. II: Time evolution of the radius $R$ (solid line) and the "center of energy" radius $R_{E}$ (dashed line) during the collapse of the circular global string loop in our largest 2-dim. simulation. $R_{0}=900, L / 2=1500$ and $\lambda=0.125$.

Fig. III: The ratio $T / R_{0}$, where $T$ is the collapse time and $R_{0}$ the initial radius of a collapsing circular loop, as a function of $\ell n\left(R_{0} / \delta\right)$. The solid (empty) triangles are from 2-dim. (3-dim.) simulations. The meaning of the solid and dashed curves is explained in the text.

Fig. IV. Energy spectrum before (white histogram) and after (black histogram) the collapse of a circular global string loop of initial radius $R_{0}=40$. The energy in the zero mode is shown in the $\log k=-\infty$ bin. The bin containing the lowest non-zero modes has arbitrarily been labeled $\log k=3$.

Fig. V: Time evolution of $N_{a x}$ during the collapse of circular global string loops of initial radii $R_{0}=40$ (solid curve) and $R_{0}=20$ (dashed curve). $N_{a x}$ is normalized to its initial value. Time $t$ is normalized to the collapse time $T$.

Fig. VI: Amplitude of an oscillating bent string as a function of time, for two different initial amplitudes. For both simulations, $L_{x} L_{y} L_{z}=240 \times 240 \times 74$ and $\lambda=0.5$. The wavelength of the bent in the string equals $L_{z}$ in both cases.

Fig. VII: Amplitude of an oscillating bent string as a function of time from three simulations 
whose initial conditions are approximatel related by a scale transformation:

$$
\begin{aligned}
L_{x} L_{y} L_{z} & =100 \times 100 \times 34, \quad A_{0}=7.0 \quad(\text { dotted line }) \\
& =170 \times 170 \times 62, \quad=12.1 \quad(\text { dashed line }) \\
& =300 \times 300 \times 114, \quad=21 \quad(\text { solid line })
\end{aligned}
$$

To facilitate comparison, the amplitude is normalized to the initial amplitude and time is normalized to the wavelength $\Lambda$. In all three cases, $\Lambda=L_{z}$ and $\lambda=0.5$.

Fig. VIII: The spectrum of a straight global string $\left(A_{0}=0\right)$. The box size is $L_{x} L_{y} L_{z}=4096 \times$ $4096 \times 1 . \lambda=0.5$. The zero mode $(\mathrm{k}=0)$ bin is not shown.

Fig. IX: The spectra of static bent strings of amplitude $A_{0}=0$ (dashed), $A_{0}=13$ (black) and $A_{0}=26$ (white). The box size is $(128)^{3}$ and $\lambda=0.5$ in all three cases.

Fig. X: The time evolution of $N_{a x}$ for two oscillating bent string simulations with initial amplitudes $A_{0}=7.5$ and $A_{0}=15$ in a box of size $(64)^{3}$. In both cases $\Lambda=64$ and $\lambda=0.5$.

Fig. XI: The time evolution of $N_{a x}$ for two oscillating bent string simulations with initial amplitudes $A_{0}=15$ and $A_{0}=30$ in a box of size (128) ${ }^{3}$. In both cases $\Lambda=128$ and $\lambda=0.5$.

Fig. XII: Spectrum of NG radiation (black histogram) emitted by an oscillating bent string of wavelength $\Lambda=64$ and initial amplitude $A_{0}=15 . \lambda=0.5$. The white histogram shows the difference of the static field energy spectra of the string when bent $\left(A_{0}=15\right)$ and when straight $\left(A_{0}=0\right)$. 


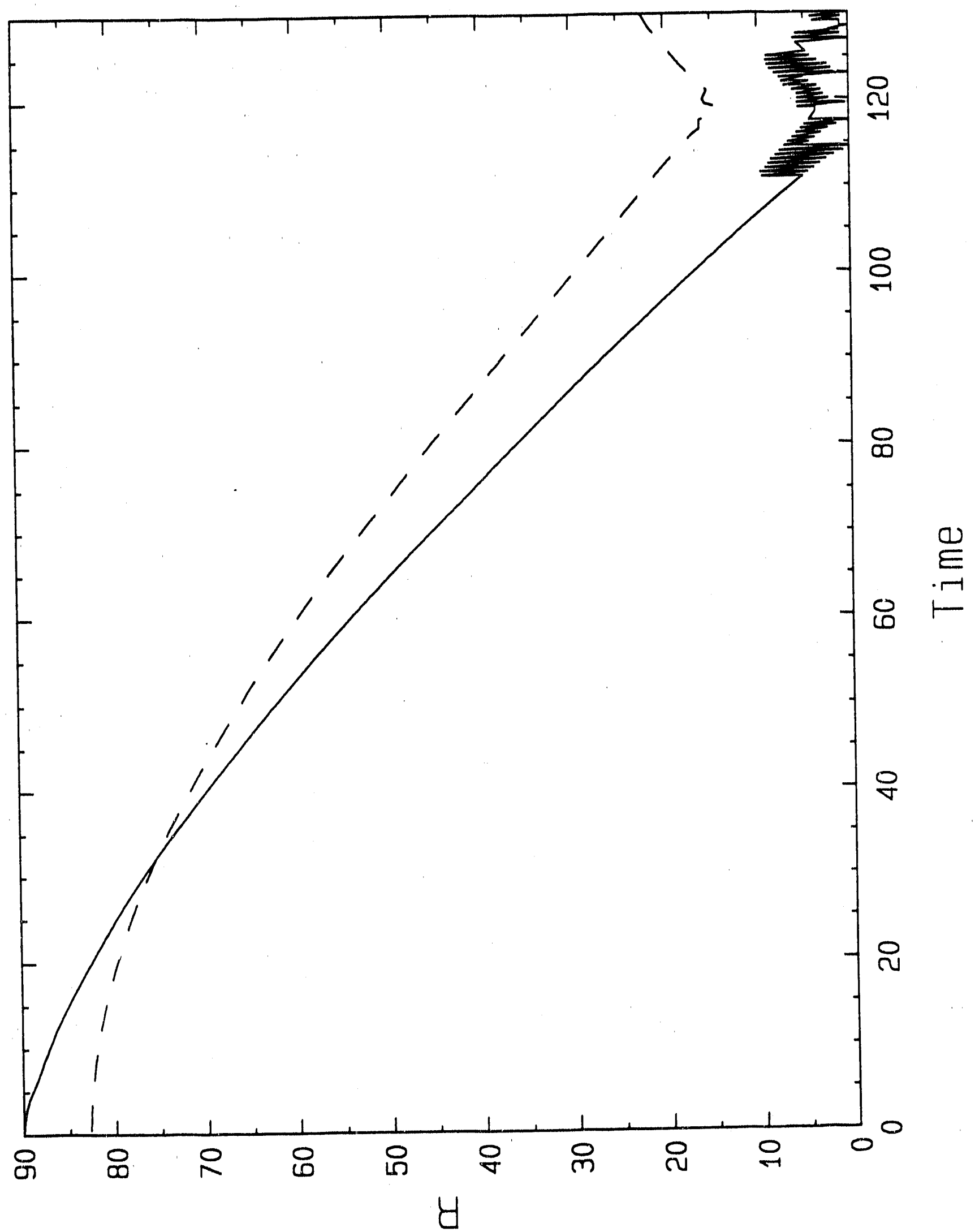

Figure I 


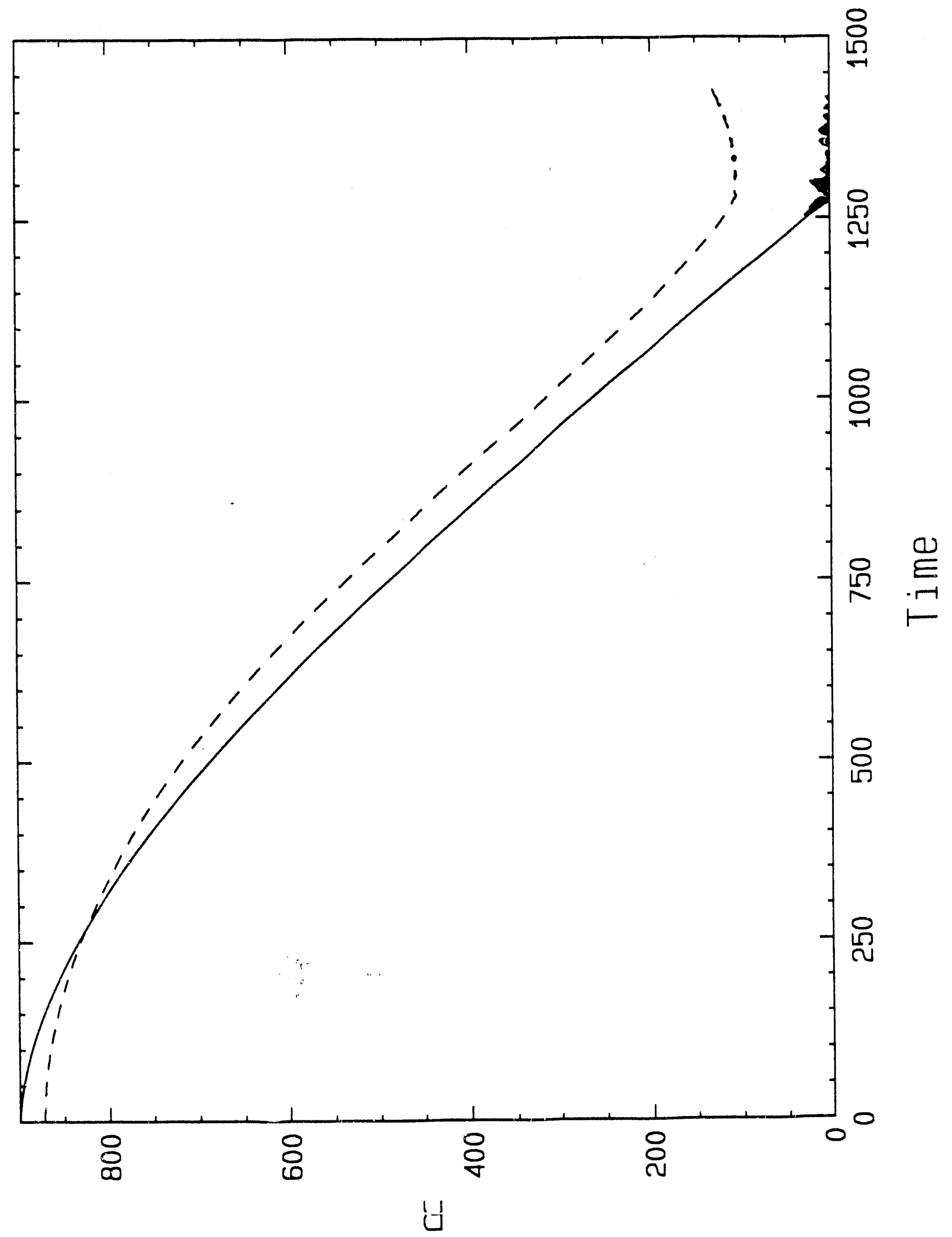

Figure II 


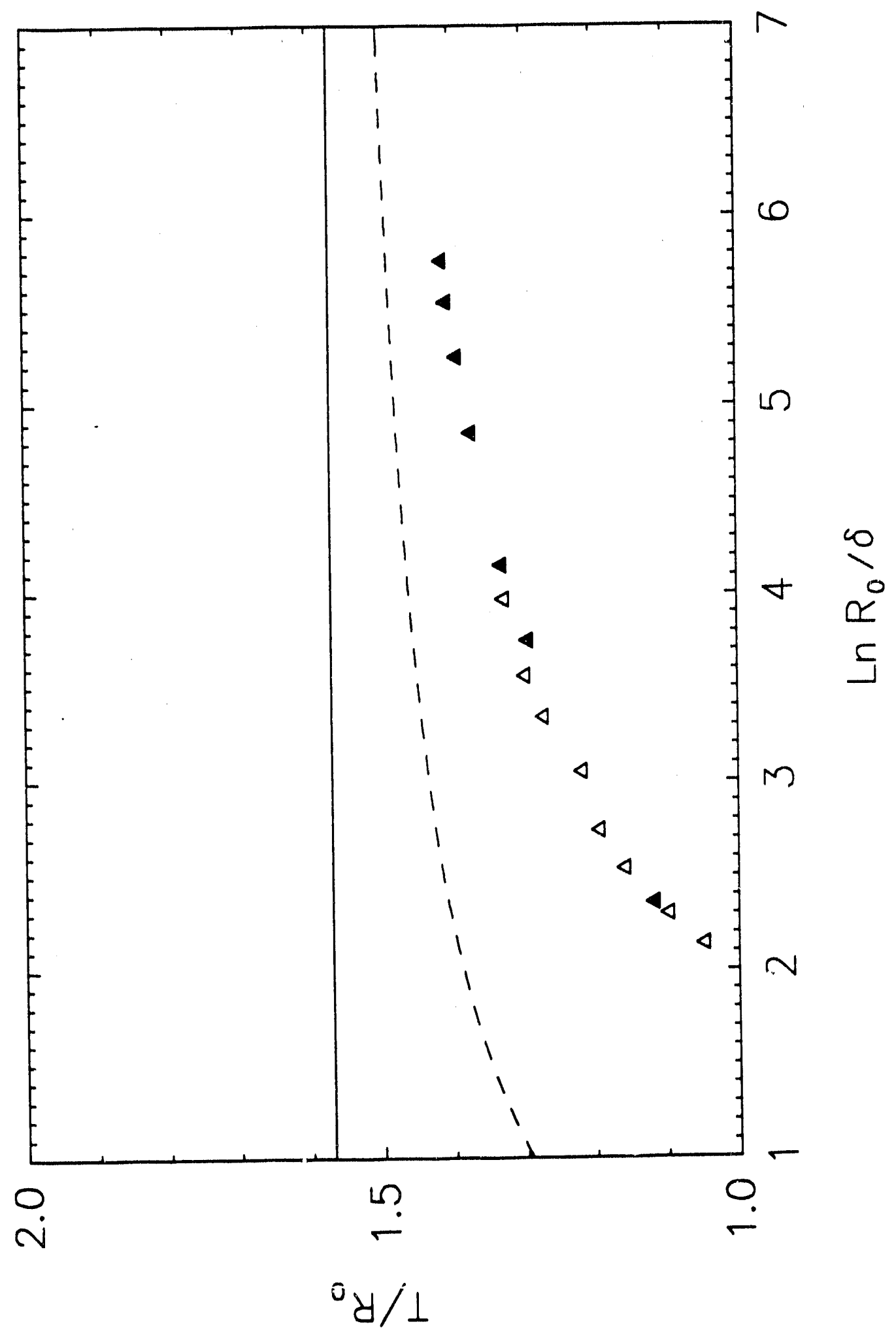

Figure III 


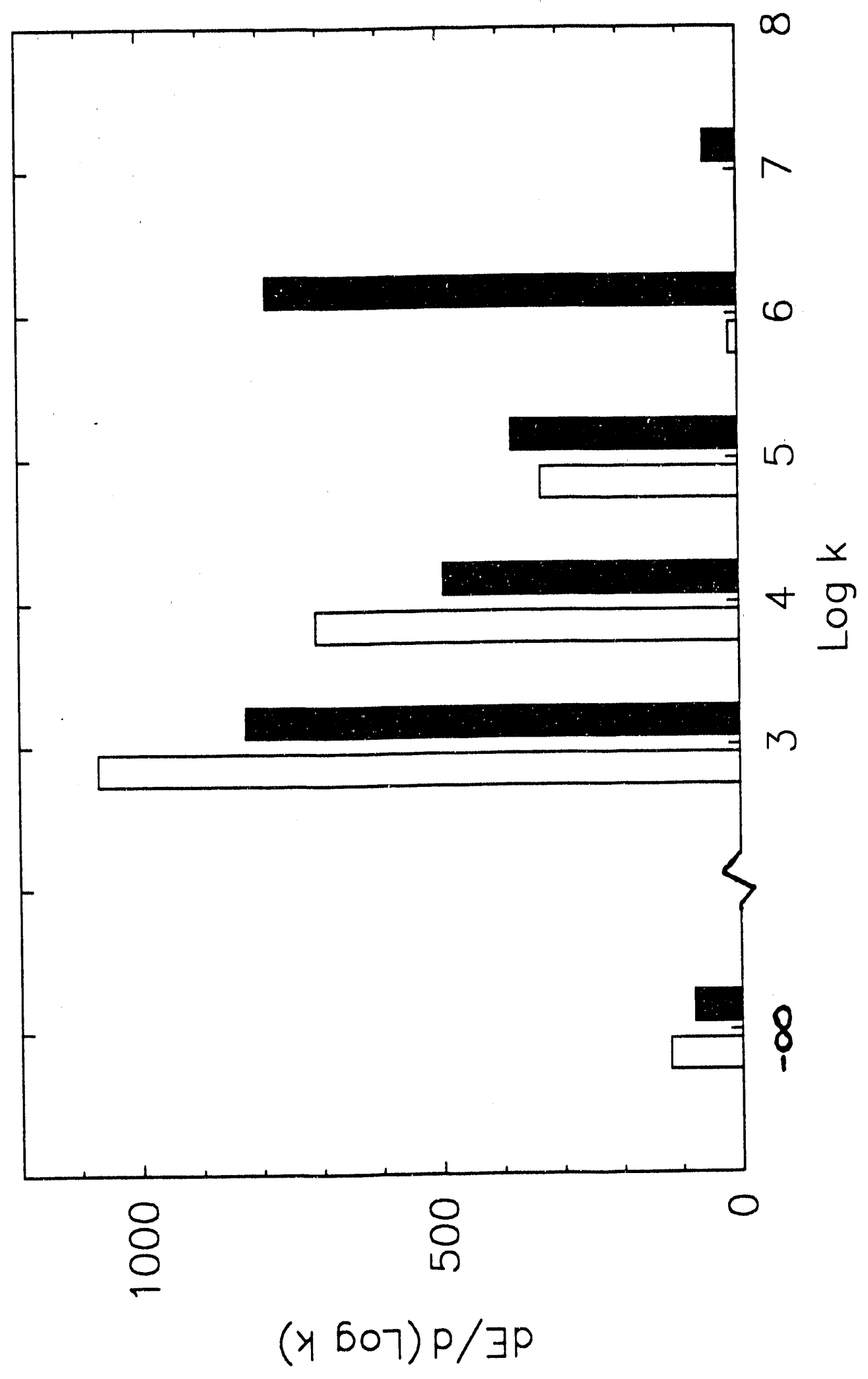

Figure IV 


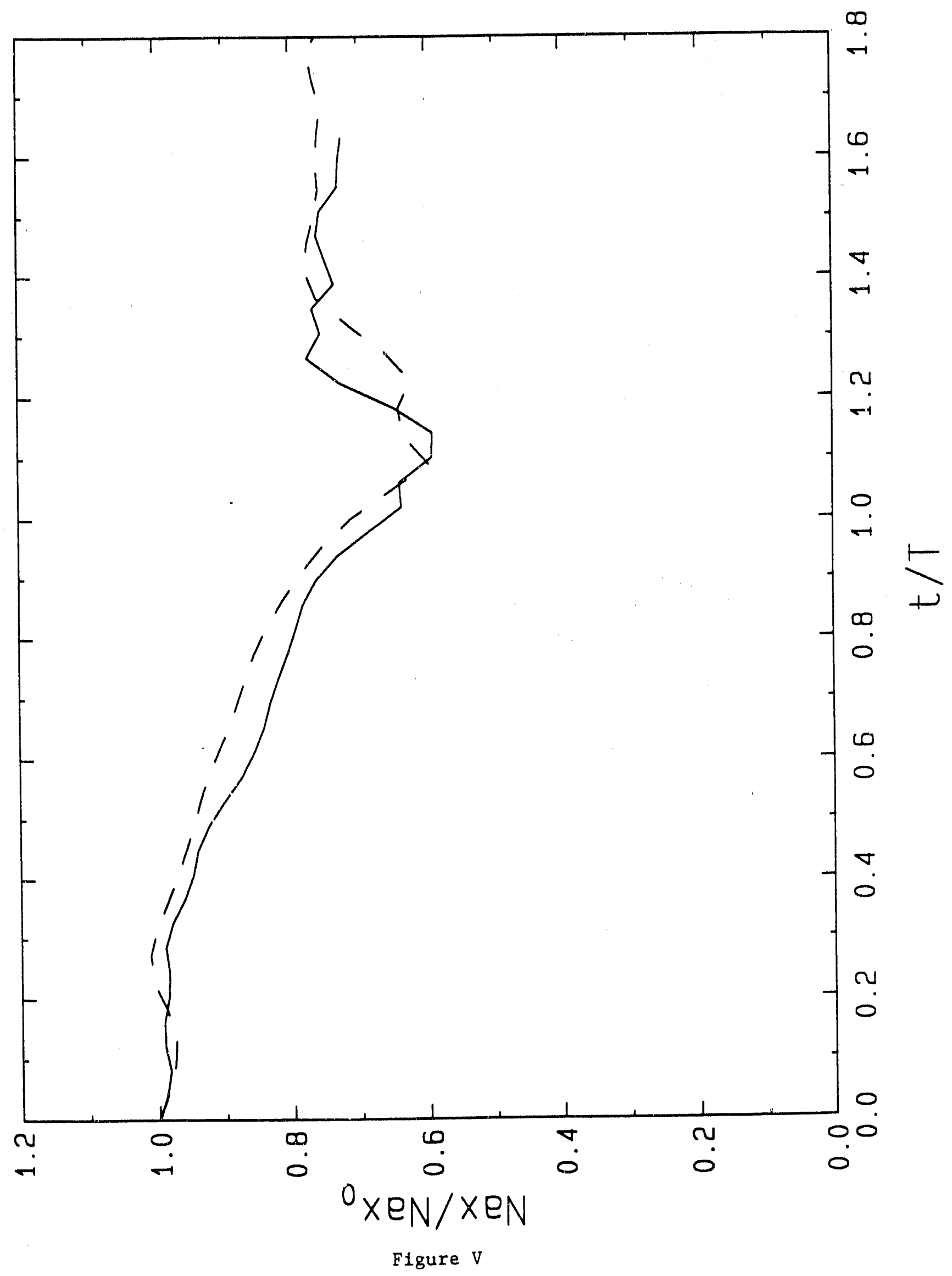




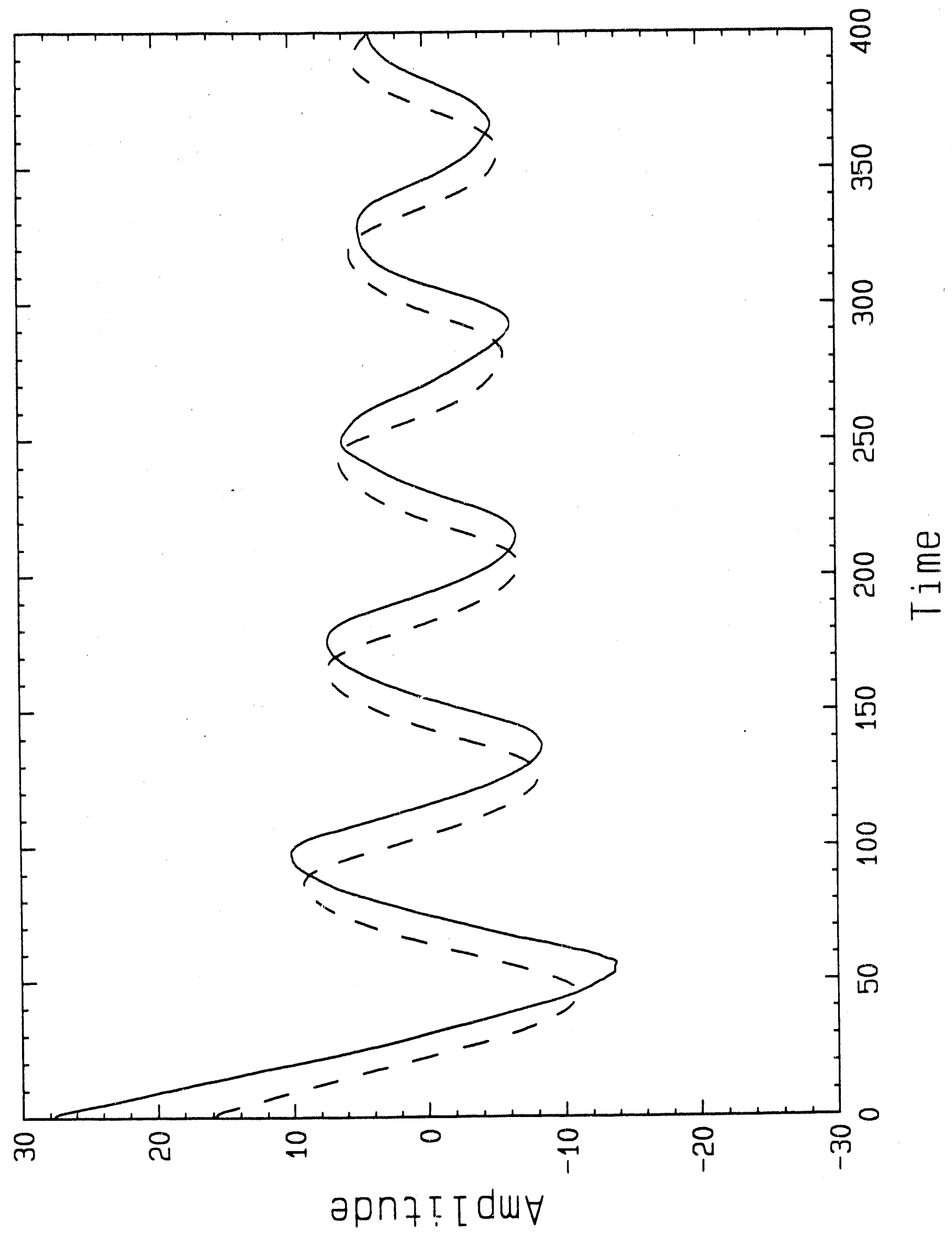

Figure VI 


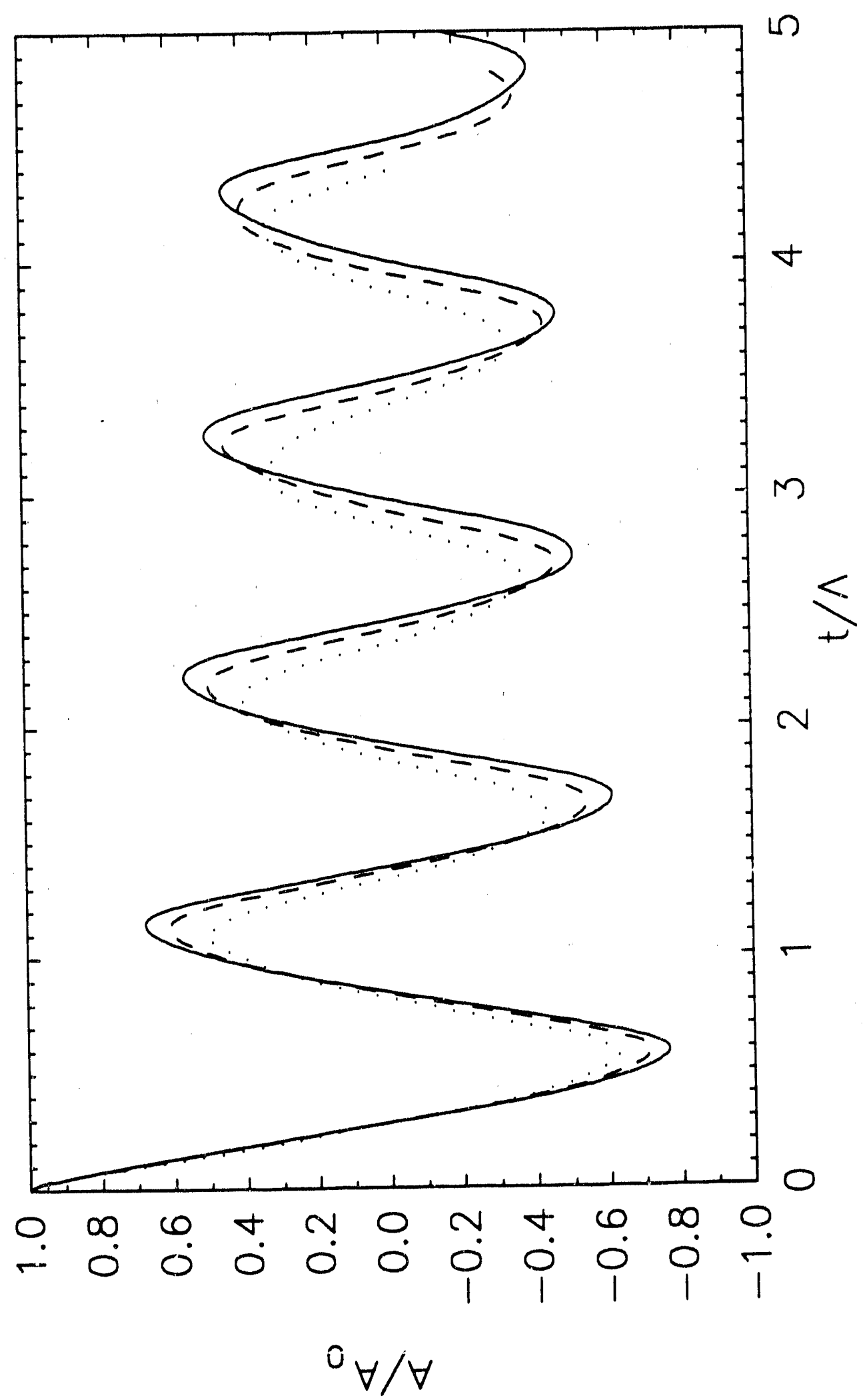

Figure VII 


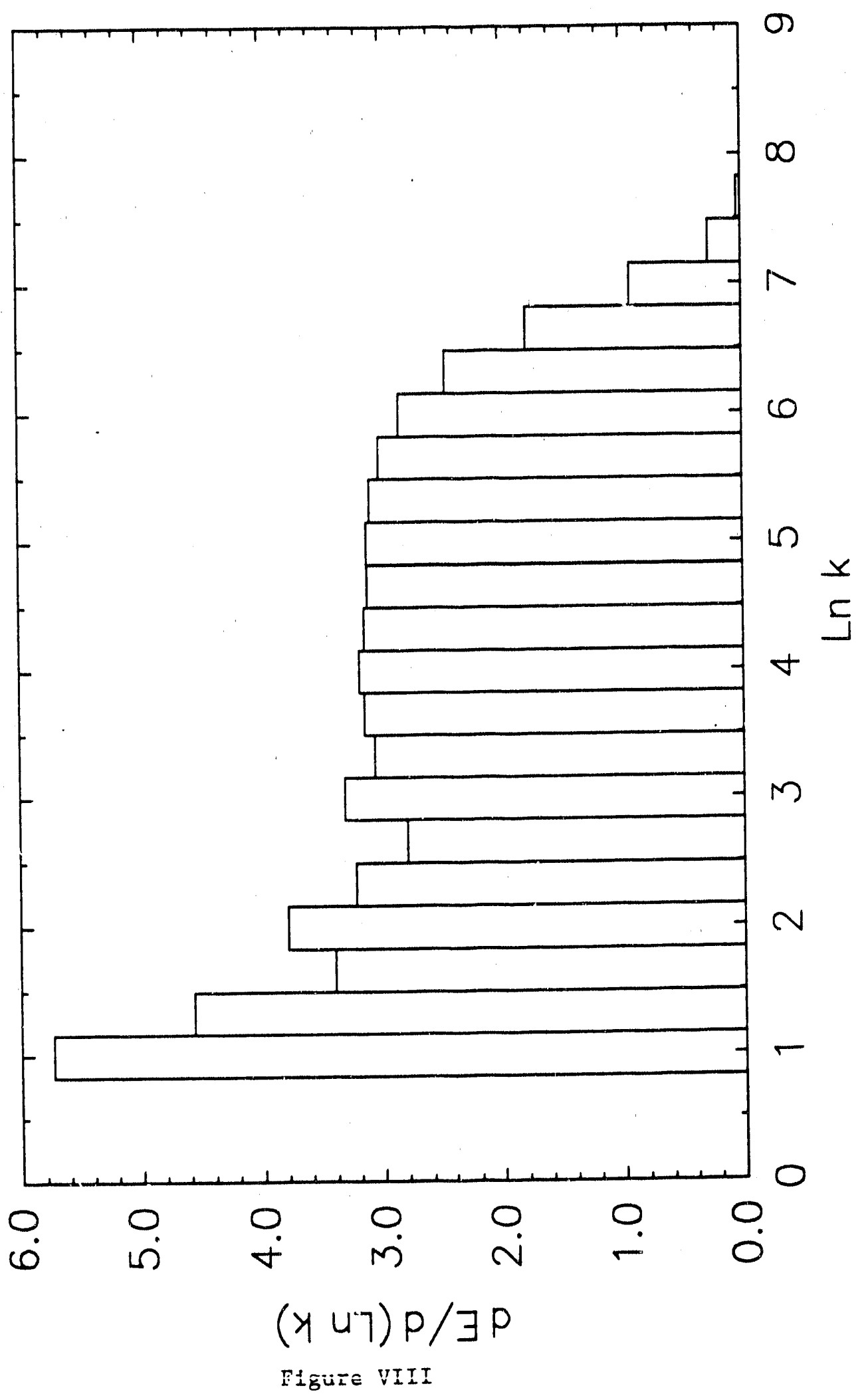




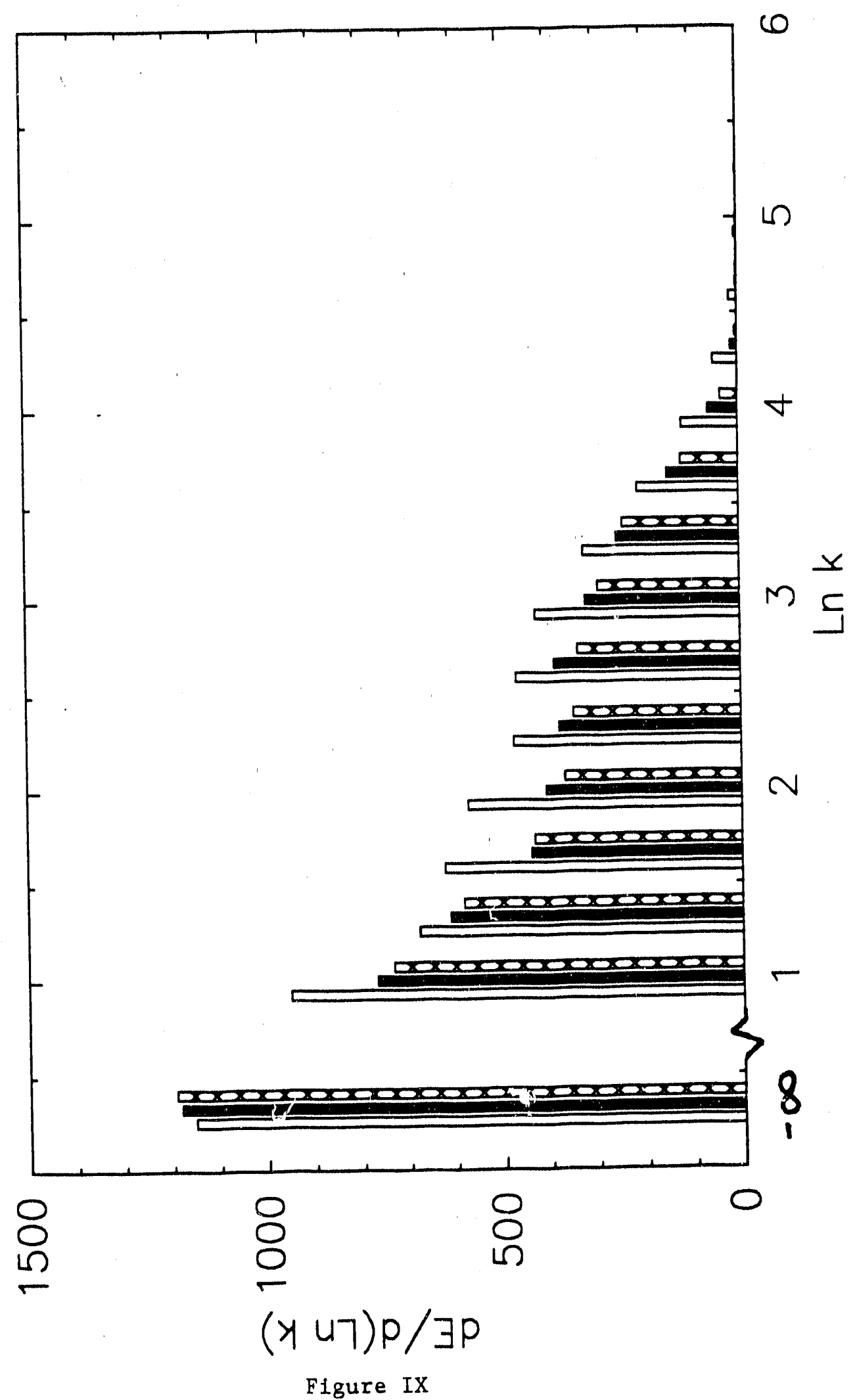




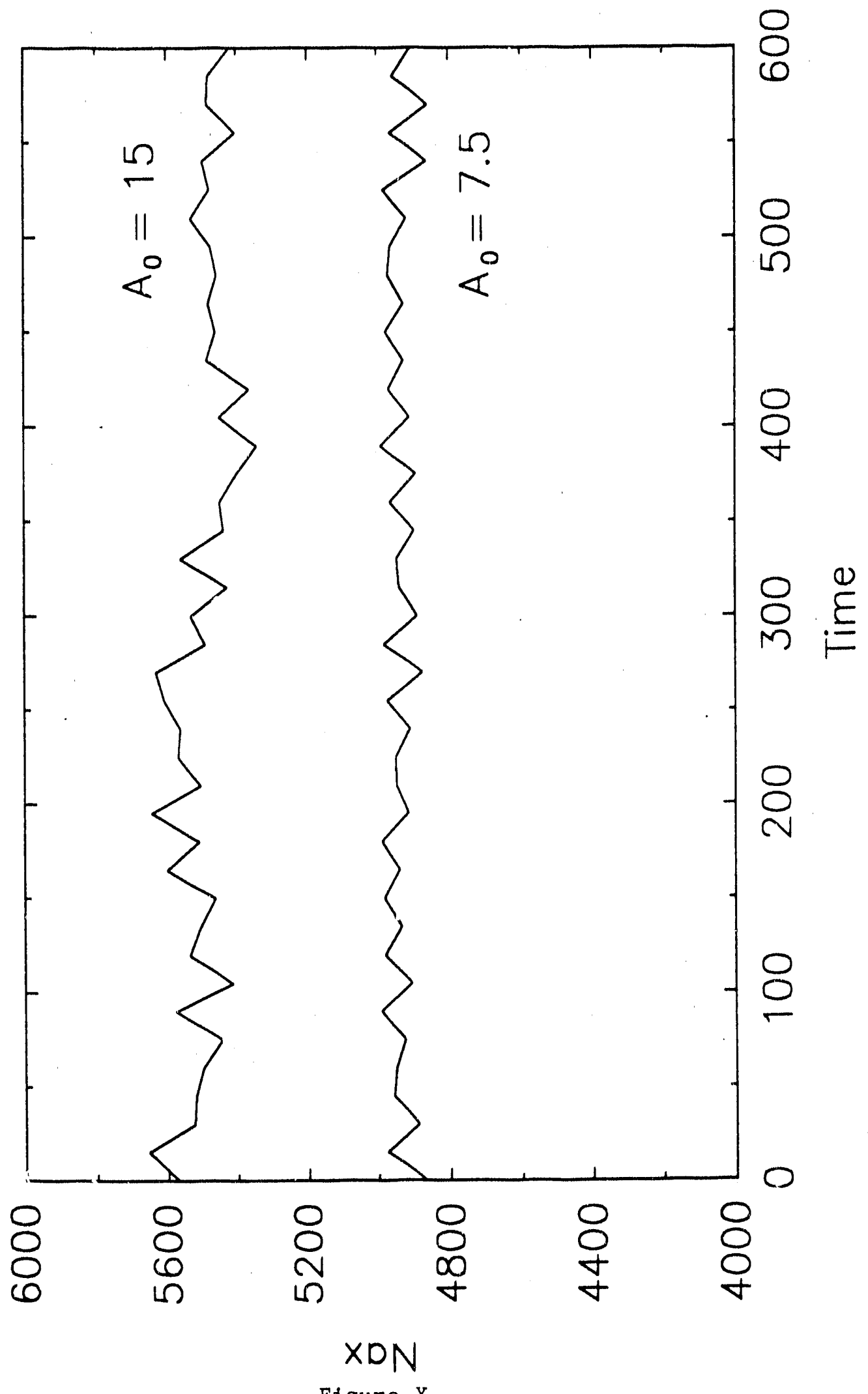

Figure $X$ 


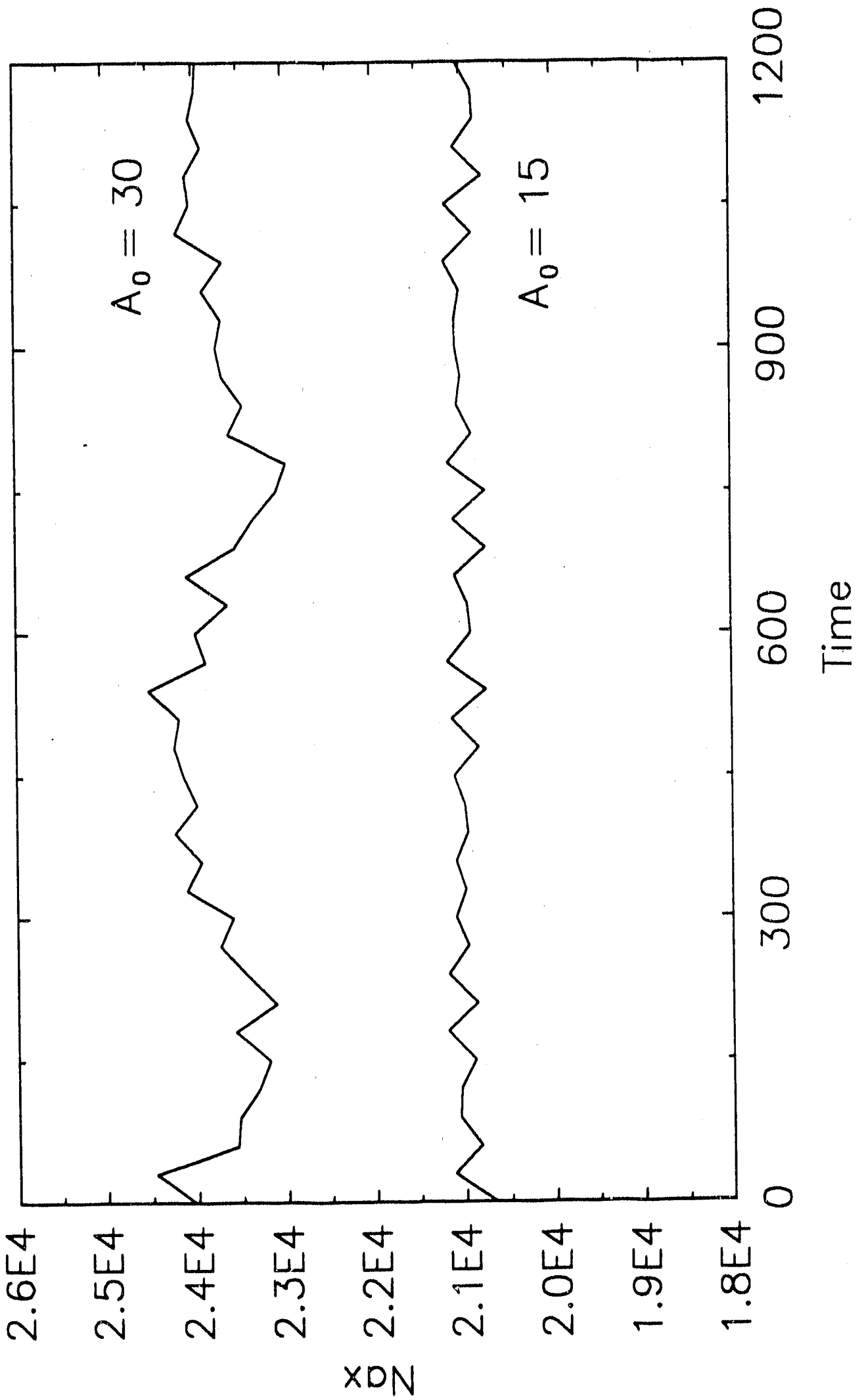

Figure XI 


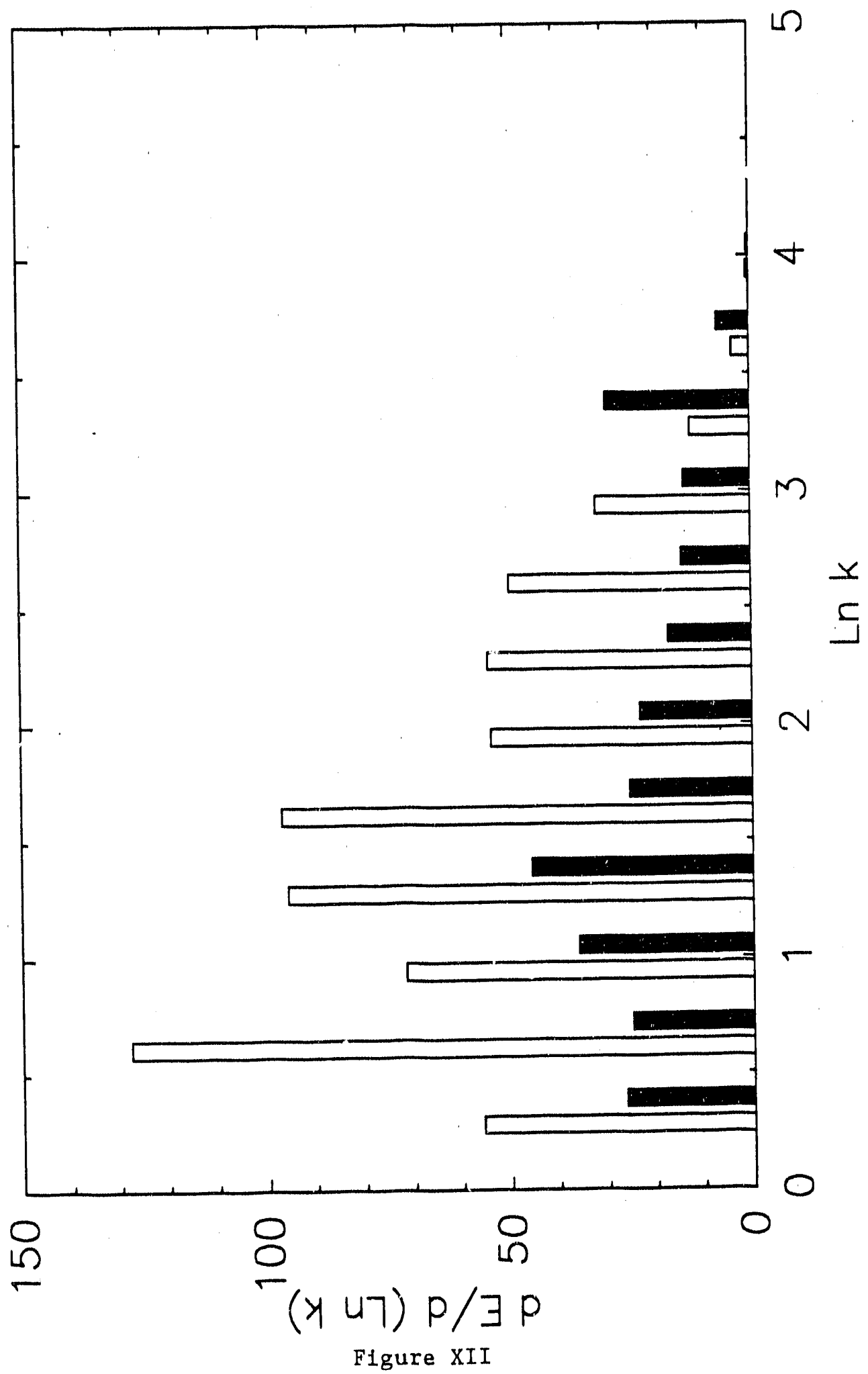



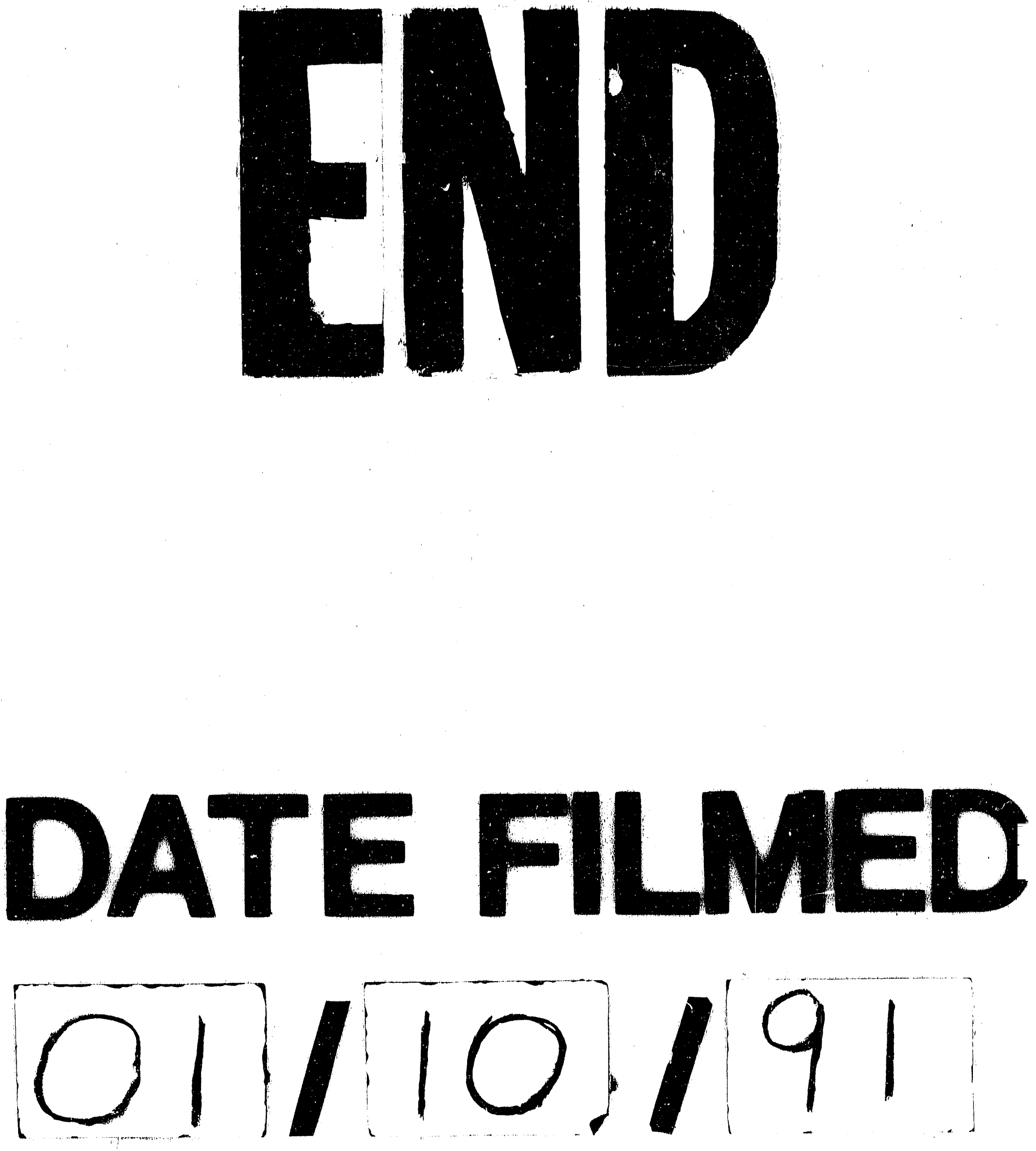
